\title{
Two-phase modeling of turbulence in dilute sediment-laden, open-channel flows
}

\author{
Sanjeev K. Jha · Fabián A. Bombardelli
}

Received: 17 February 2008 / Accepted: 5 December 2008 / Published online: 8 January 2009

(C) The Author(s) 2009. This article is published with open access at Springerlink.com

\begin{abstract}
In this paper, we focus on assessing the performance of diverse turbulence closures in the simulation of dilute sediment-laden, open-channel flows. To that end, we base our analysis on a framework developed in a companion paper of this special issue, which puts forward a standard sediment transport model (SSTM), a partial two-fluid model (PTFM) and a complete two-fluid model (CTFM), in three- and one-dimensional (3D and 1D) versions. First, we propose in this paper extensions of the transport equations for the Reynolds stresses, and of the equations of the $K-\omega$ model to two-phase flows, starting from the general two-fluid model. We consider the drag force to be the predominant force amongst all the interactions between the two phases (water and sediment). Second, under the framework of models formed by the SSTM, the PTFM and the CTFM, we discuss simulation results obtained by employing the Reynolds stress model (RSM), the algebraic stress model (ASM), and the $K-\varepsilon$ and the $K-\omega$ models (in their standard and extended versions), paired with each member of the framework. To assess the accuracy of the models, we compare numerical results with the experimental datasets of Vanoni, Trans ASCE 111:67-133, 1946; Coleman, Water Resour Res 22(10):1377-1384, 1986; Muste and Patel, J Hydraul Eng 123(9):742751, 1997; Nezu and Azuma, J Hydraul Eng 130:988-1001, 2004; Muste et al. Water Resour Res 41:W10402, 2005. Third, we obtain from those comparisons the values of the Schmidt number that facilitate the agreement of model predictions with data. We conclude that the standard $K-\varepsilon$ model, the ASM and the $K-\omega$ models all provide satisfactory descriptions of flow variables and sediment concentrations in open-channel flows; further, we show that the more complicated RSM does not provide much improvement in dilute sediment transport as compared to those previous models, even when it is paired with the CTFM. We also show that the inclusion of model extensions in the turbulence closures does not improve the predictions for dilute mixtures either. We find that our values for the Schmidt number agree well with
\end{abstract}

S. K. Jha · F. A. Bombardelli $(\bowtie)$

Department of Civil and Environmental Engineering, University of California, Davis, 2001 Eng. III,

One Shields Ave., Davis, CA 95616, USA

e-mail: fabombardelli@ucdavis.edu

S. K. Jha

e-mail: skjha@ucdavis.edu 
available data, and we provide an explanation for the variation of the Schmidt number with the ratio of the fall velocity and the wall-friction (shear) velocity. Finally, we corroborate that the Schmidt number is the key parameter to obtain satisfactory predictions of sediment transport in suspension.

Keywords Turbulence - Reynolds stress model $\cdot$ Sediment transport - Two-phase flows · $K-\varepsilon$ model $\cdot K-\omega$ model $\cdot$ Algebraic stress model $\cdot$ RSM $\cdot$ ASM

\section{Introduction}

In a previous paper in this special issue [6], we addressed several aspects of the modeling of sediment transport, focusing on the phase interactions at the water-depth scale for dilute flows. We proposed, for the first time to the best of our knowledge, a framework constituted by three modeling approaches involving different complexities: (1) A standard sedimenttransport model (SSTM), (2) a partial two-fluid model (PTFM), and (3) a complete two-fluid model (CTFM). While the first approach represents a relatively simple attempt to model the motion of the two phases, the last one is described by a set of equations representing the conservation of mass and momentum of each phase within a fixed elemental volume of the mixture. The main assumption used in deriving the equations for the CTFM is that both phases behave macroscopically as fluid continua [21-23]. This hypothesis is customary in particle-laden flows in open channels (see for instance [11-13,20,31,30,32,34,37,49,67, 70]). By posing the SSTM, the PTFM and the CTFM in one dimension (1D) in the wall-normal direction, we were able to test in the companion paper the accuracy of the models against data by Lyn [45], Muste and Patel [50], and Muste et al. [51], in terms of the prediction of the turbulence-averaged water velocity, the averaged velocity of the disperse phase and flow turbulence statistics. We tested the effect of neglecting the eddy viscosity of the disperse phase (the sediment); we considered diverse models for the Schmidt number; and we reported those values of the Schmidt number that best approximated the data. We additionally tested the importance of certain terms added by some authors to the $K-\varepsilon$ model (the model used to treat the turbulence of the carrier phase, where $K$ and $\varepsilon$ denote the turbulent kinetic energy (TKE) and the dissipation rate of turbulent kinetic energy (DTKE), respectively), concluding that those terms do not produce major changes in the predictions in any of the framework approaches described above. These framework models were tested with just one possible turbulence closure (the $K-\varepsilon$ model); there is naturally a need to rigorously test them with other turbulence closures. Turbulence in open-channel flows is non-homogeneous and non-isotropic [5,27-29,52,57], which puts forward an important question on the choice of the most appropriate turbulence closure for modeling when the Reynolds-Averaged Navier-Stokes (RANS) equations are used. This issue is still relevant in spite of the wide use of the $K-\varepsilon$ model. In addition, the use of the Reynolds stress model (RSM) in two-phase flows has been scarce, and reasonable questions arise as to which values of the vertical component of the velocity of the disperse phase and the Schmidt number such closure would yield when paired with the CTFM. Finally, Choi and Kang [15] found that the RSM improved the description of most flow variables in open channels with vegetation (in 1D), as opposed to the $K-\varepsilon$ model. Thus, a fair issue surfaces as to whether the application of the RSM to sediment-laden, open-channel flows can yield improvement in the model predictions. We keep our focus on dilute flows. In the case of dilute flows, the volume fraction of sediment is small ( $\alpha_{d} \approx 10^{-3}$ to $10^{-2}$ as a rough estimate [22]). 
The dilute flow is defined as the flow in which particle-particle interactions can be neglected $[32,44]$.

Based on the above concepts, this paper evaluates the effect of various turbulence closures in the simulation of open-channel, sediment transport, under different approaches of modeling discussed in [6]. To the best of our knowledge, this is the first time that the focus on modeling in sediment transport engineering is shifted completely to address the performance of different turbulence closures against common datasets (cf. [46]).

The paper is organized as follows. In Sect.2, we describe very recent contributions on the turbulence modeling in single- and two-phase flows using the RSM, the algebraic stress model (ASM) and the $K-\varepsilon$ and $K-\omega$ models (where $\omega$ denotes the specific dissipation rate of TKE per unity TKE; see [72]). In Sect.3, we briefly review the developed framework of models for dilute sediment-laden, open-channel flows presented in [6], and discuss single-phase turbulence models. In Sect. 4, we introduce the extensions of the RSM and the $K-\omega$ model to two-phase flows. We present the group of numerical tests that we performed in Sect.5, and we discuss the comparisons between those results and data in Sect.6, for both mean-flow variables, flow turbulence statistics, and for sediment distributions.

\section{Some recent contributions on turbulence closures}

Although based on the isotropic eddy-viscosity concept, the $K-\varepsilon$ model has been the most widely used model in single-phase flows. This model has provided accurate results in many cases (see [58]) for mean-flow variables and turbulence statistics. However, the model has been found to be not completely adequate for solving certain aspects of highly anisotropic flows such as the flows in compound channels, meandering channels in which curvature effects are important, vegetated channels, and swirling flows; in those cases the RSM becomes a more accurate tool $[15,16,35,59,63,75]$. The RSM has been recently shown to improve also the predictions of shear stresses and some turbulence statistics in wall-bounded flows [33] and open-channel flows with vegetation [15]. The ASM, in turn, involves algebraic expressions for the Reynolds stresses obtained by simplifying the terms in the transport equations of the RSM (see for example [25,56]). Another turbulence closure based on the eddy-viscosity concept is the $K-\omega$ model [72]. The specific dissipation rate $(\omega)$ replaces the DTKE $(\varepsilon)$ in defining the flow length scale.

Concerning the application of these closures to two-phase flows in general, and sediment transport in particular, the knowledge is not that clear. Only few studies, to the best of our knowledge, have addressed the influence of different turbulence closures on the prediction of the main variables in the flow, and not much is known about the relative or marginal improvement that the use of these models could provide under diverse situations. Most of the available information for two-phase flows comes from bubbly flows (see for instance $[4,61,62])$. Furthermore, we have not been successful in finding any paper for sedimentladen flows addressing the relation between the turbulence closure on one hand and the level of complexity of the sub-models dealing with the two phases on the other (i.e., the way the carrier and the disperse phases are described). Cao and Carling [10] estimated that probably the use of second-order closures (RSM) for sediment transport would not provide much improvement over the $K-\varepsilon$ model, involving instead a higher computational effort. However, they neither presented computations attesting to this fact nor discussed which aspects of the prediction would be improved using the RSM. 
$2.1 K-\varepsilon$ model

Elghobashi and Abou-Arab [23] presented one of the first rigorous derivations of the twophase $K-\varepsilon$ model. While their generalized transport equation for $K$ contains 38 terms, the transport equation for $\varepsilon$ contains 59 terms, which Elghobashi and Abou-Arab finally reduced to 11 and 7 terms, respectively. Elghobashi and Abou-Arab employed the model to simulate dilute, particle-laden jet flows. On similar grounds, Hsu et al. [32] derived a two-phase $K-\varepsilon$ model by disregarding several correlations. They reported an improvement in their predictions of dilute, sediment-laden, open-channel flows. Zhou and Chen [77], on the other hand, proposed a closure for turbulence in the carrier as well as the disperse phases starting from the equations of the Reynolds stresses of the two phases. This model uses a transport equation for the particle turbulent kinetic energy $\left(K_{p}\right)$ in addition to the transport equations for $K$ and $\varepsilon$. Zhou and Chen employed their model to analyze swirling, gas-particle flows. Mashayek and Taulbee [48] proposed a $K-\varepsilon-K_{p}-K_{f p}$ model, which uses an additional transport equation for the velocity correlation of the two phases $\left(K_{f p}\right)$. Mashayek and Taulbee derived these equations by contracting the equations for the fluid Reynolds stresses, the particle Reynolds stresses, and the covariance between the fluctuations of both phases. Mashayek and Taulbee applied their model to particle-laden, turbulent jets with encouraging results. López and García [42] applied a $K-\varepsilon$ model to assess the turbulence characteristics and the transport of suspended sediment in open channels through emergent vegetation. López and García added a drag related term in the transport equations for $K$ and $\varepsilon$ due to vegetation and concluded that this form of $K-\varepsilon$ model becomes a reliable tool to predict the sediment transport under different flow, sediment and vegetation conditions.

\section{$2.2 K-\omega$ model}

López and García [43] applied both the $K-\varepsilon$ and $K-\omega$ models for the simulation of flow through emergent vegetation. They found no significant difference in numerical results using both closures. Yoon and Kang [74] introduced a slight modification to the standard Wilcox's [72] $K-\omega$ model in order to simulate the flow of particles in water. While the transport equations for $K$ and $\omega$ remained the same as in the standard Wilcox's model, they used a new eddy viscosity through which they included the effect of suspended sediment on the flow.

\subsection{RSM}

Bertodano et al. [3] used the RSM for the carrier phase turbulence in the case of turbulent bubbly flows. de Bertodano et al. considered the lift force as the most important interaction force in their work. Taulbee et al. [66] and Lain and Aliod [39] developed a RSM for both phases, continuous and disperse, and considered the drag force as the predominant force between the two phases (also see [1,18]). Sijercic et al. [60] used the RSM (in its single-phase form) in the simulation of particle-laden, turbulent jets injected into a still environment. In their analysis, Sijercic et al. did not include the turbulence modulation due to the presence of the disperse phase.

\subsection{ASM}

Kumar [38] introduced an ASM for two-phase, bubbly and slug turbulent flows, by modifying the expressions for the Reynolds stresses. The resulting model has additional constants, but Kumar did not pursue the validation of the model against data. 
These studies refer mostly to bubbly flows and gas-solid particle flows; in gas-solid flows, the ratio of the densities of the two phases is large. In the case of sediment-laden, water flows, the ratio of densities is order 1 (it varies between 1.5 and 3). Thus, those results should in principle be extrapolated with caution to water-sediment flows. Consequently, there is a natural uncertainty as to whether the closures can work fine in sediment-laden, water flows as well. As a general conclusion, not much research has been devoted to the analysis and testing of two-phase versions of the $K-\omega$ model, and the RSM.

\section{Framework for modeling sediment-laden, open-channel flows and turbulence closures for single-phase flows}

\subsection{Framework for the simulation of sediment-laden, open-channel flows}

Bombardelli and Jha [6] defined three levels of complexity in modeling sediment transport in open channels. The most complex model in the framework, termed as the CTFM, is composed by general balance equations of mass and momentum for the two phases, which can be reduced in length by considering only the drag force as the most important interaction between the phases. Unlike other CTFM used in the past, our model equations involve both an ensemble and turbulence averaging, in order to obtain a diffusion term in the mass balance equations (see [4] and [9]). The final form of the mass and momentum equations [6,7,21] can be found in Table 1 .

In Eqs. T1 and T2, the subscript $k$ stands for both phases (which could be $c$ or $d$ for the carrier and the disperse phases, respectively); $\alpha_{k}$ represents the volume fraction of the phase $k$ [21, p. 122] which is a turbulence-averaged variable; $\rho_{k}$ is the density of phase $k$; and $U_{k}$ represents the velocity of phase $k$, which is also an ensemble- and turbulence-averaged variable. In turn, $\Gamma_{k}$ is the mass transfer to the phase $k$ (which is zero in this case); $P$ is the pressure; $T_{i j}$ refers to the deviatoric stresses; the stresses with the superscript $R e$ are the result of the processes of ensemble and turbulence averaging; $g_{i}$ is the $i$-th component of the acceleration due to gravity; and $x$ and $t$ are the space and time coordinates, respectively. The indices $i$ and $j$ vary from 1 to 3 and sum is implied in repeated indices. Bombardelli [4] showed that the momentum equation of phase $k$ becomes very involved when all correlations are considered. Some terms include triple correlations for which very little is known. Therefore, the format of Eq. T2 represents a good compromise between simplicity and rigor. Equations T1 and T2 involve in fact four equations in total. The first and second terms on the left-hand side of Eq. T1 are the rate of change of mass in a point and the net rate of convective mass flux. The term on the right hand side of Eq. T1 represents the transport by molecular and turbulent diffusion. The first and second terms on the left-hand side of Eq. T2 are the net rate of change of momentum in a point and the net rate of momentum transfer by convection, respectively; the first term on the right-hand side is the mean pressure gradient; the second term on the right-hand side is the stress term; the third term on the right-hand side includes the body forces such as gravity.

Stresses are modeled as $T_{i j, k}^{R e}=-\rho_{k} \overline{u_{i, k}^{\prime} u_{j, k}^{\prime}}$, where the superscript ' indicates fluctuation $[4,9,21]$. The last term in Eq. T2 represents the drag force, involving $\beta=\frac{3}{4 d_{p}} \alpha_{d} \rho_{c} C_{D}\left|\vec{U}_{r}\right|$, where the subscript $r$ indicates the relative velocity between the two phases; $d_{p}$ is the diameter of the sediment particles; and $C_{D}$ is the drag coefficient, defined in the companion paper.

The second and somewhat less-involved model is termed as the PTFM, which consists of mixture equations as a surrogate of the carrier phase and general equations for the dilute 
Table 1 Two-phase flow equations

\section{CT F M}

Mass balance

$\frac{\partial\left(\alpha_{k} \rho_{k}\right)}{\partial t}+\frac{\partial}{\partial x_{j}}\left(\alpha_{k} \rho_{k} U_{j, k}\right)=\frac{\partial}{\partial x_{j}}\left(\rho_{k} \overline{\alpha_{k}^{\prime} u_{j, k}^{\prime}}\right)+\Gamma_{k}$

Momentum balance

$\frac{\partial\left(\alpha_{k} \rho_{k} U_{i, k}\right)}{\partial t}+\frac{\partial}{\partial x_{j}}\left(\alpha_{k} \rho_{k} U_{i, k} U_{j, k}\right)=-\alpha_{k} \frac{\partial P_{k}}{\partial x_{i}}+\frac{\partial}{\partial x_{j}}\left[\alpha_{k}\left(T_{i j, k}+T_{i j, k}^{R e}\right)\right]+\alpha_{k} \rho_{k} \mathrm{~g}_{i} \pm \beta U_{i, r}$

Mass balance for the carrier

$\frac{\partial}{\partial x_{j}}\left(U_{j, m}\right)=0$

Momentum balance for the carrier

$\frac{\partial\left(\rho_{0} U_{i, m}\right)}{\partial t}+\frac{\partial}{\partial x_{j}}\left(\rho_{0} U_{i, m} U_{j, m}\right)=-\frac{\partial P_{m}}{\partial x_{i}}+\frac{\partial}{\partial x_{j}}\left(T_{i j, m}+T_{i j, m}^{R e}\right)+\rho_{m} g_{i}$

Mass balance for the disperse phase

$\frac{\partial\left(\alpha_{d} \rho_{d}\right)}{\partial t}+\frac{\partial}{\partial x_{j}}\left(\alpha_{d} \rho_{d} U_{j, d}\right)=-\frac{\partial}{\partial x_{j}}\left[\rho_{d} \overline{\alpha_{d}^{\prime} u_{j, d}^{\prime}}\right]$

Momentum balance for the disperse phase

$\frac{\partial\left(\alpha_{d} \rho_{d} U_{i, d}\right)}{\partial t}+\frac{\partial}{\partial x_{j}}\left(\alpha_{d} \rho_{d} U_{i, d} U_{j, d}\right)=-\alpha_{d} \frac{\partial P_{c}}{\partial x_{i}}+\frac{\partial}{\partial x_{j}}\left[\alpha_{d}\left(T_{i j, d}+T_{i j, d}^{R e}\right)\right]+\alpha_{d} \rho_{d} g_{i}+\beta U_{i, r}$ SSTM

Advection-diffusion equation to calculate the distribution of sediment concentration

$\frac{\partial C}{\partial t}+\frac{\partial}{\partial x_{j}}\left[C\left(U_{j, d}-W_{s} \delta_{j 3}\right)\right]=-\frac{\partial}{\partial x_{j}}\left[\overline{c^{\prime} u_{j, d}^{\prime}}\right]$

Transport equations of the RSM for single-phase flows

$\frac{D}{D t}\left[\rho\left(\overline{u_{i}^{\prime} u_{j}^{\prime}}\right)\right]=P_{i j}+D_{i j}+\pi_{i j}-\varepsilon_{i j}$

$P_{i j} \equiv \rho\left\{-\left(\overline{u_{i}^{\prime} u_{l}^{\prime}}\right) \frac{\partial U_{j}}{\partial x_{l}}-\left(\overline{u_{j}^{\prime} u_{l}^{\prime}}\right) \frac{\partial U_{i}}{\partial x_{l}}\right\} ; D_{i j} \equiv C_{S} \frac{\partial}{\partial x_{l}}\left[\rho\left\{\frac{K}{\varepsilon} \overline{u_{l}^{\prime} u_{q}^{\prime}} \frac{\partial}{\partial x_{q}}\left(\overline{u_{i}^{\prime} u_{j}^{\prime}}\right)\right\}\right]$

$\pi_{i j} \equiv-C_{1} \rho \frac{\varepsilon}{K}\left\{\overline{u_{i}^{\prime} u_{j}^{\prime}}-\frac{2}{3} \delta_{i j} K\right\}-C_{2} \rho\left(P_{i j}-\frac{2}{3} \delta_{i j} \operatorname{prod}\right) ; \varepsilon_{i j} \equiv \frac{2}{3} \rho \varepsilon \delta_{i j}$

Expression for the stresses in the ASM

$-\overline{u_{i}^{\prime} u_{j}^{\prime}}=K\left[\frac{P_{i j}\left(1-C_{2}\right)+\frac{2}{3} \delta_{i j}\left[\operatorname{prod} C_{2}-\left(1-C_{1}\right) \varepsilon\right]}{\operatorname{prod}-\left(1-C_{1}\right) \varepsilon}\right]$

Expression for the Reynolds stresses

$\overline{u_{i, c}^{\prime} u_{j, c}^{\prime}}=-v_{T}\left(\frac{\partial U_{i, c}}{\partial x_{j}}+\frac{\partial U_{j, c}}{\partial x_{i}}\right)+\frac{2}{3} K \delta_{i j} ; \overline{\alpha_{d}^{\prime} u_{j, d}^{\prime}}=-\frac{v_{T}}{\sigma} \frac{\partial \alpha_{d}}{\partial x_{j}}$

Expression for the Reynolds fluxes

$\overline{c^{\prime} u_{j, d}^{\prime}}=-\frac{v_{T}}{\sigma} \frac{\partial C}{\partial x_{j}}$

Transport equation for the TKE

$\frac{\partial\left(\rho_{c} K\right)}{\partial t}+U_{c, j} \frac{\partial\left(\rho_{c} K\right)}{\partial x_{j}}=\frac{\partial}{\partial x_{j}}\left[\frac{\nu_{T}}{\sigma_{K}} \frac{\partial\left(\rho_{c} K\right)}{\partial x_{j}}\right]+\operatorname{prod}-\rho_{c} \varepsilon+S_{K}$

Transport equation for the DTKE

$\frac{\partial\left(\rho_{c} \varepsilon\right)}{\partial t}+U_{c, j} \frac{\partial\left(\rho_{c} \varepsilon\right)}{\partial x_{j}}=\frac{\partial}{\partial x_{j}}\left[\frac{v_{T}}{\sigma_{\varepsilon}} \frac{\partial\left(\rho_{c} \varepsilon\right)}{\partial x_{j}}\right]+C_{\varepsilon 1} \operatorname{prod} \frac{\varepsilon}{K}-C_{\varepsilon 2} \rho_{c} \frac{\varepsilon^{2}}{K}+S_{\varepsilon}$

Transport equation for the specific dissipation $(\omega)$

$\frac{\partial\left(\rho_{c} \omega\right)}{\partial t}+U_{c, j} \frac{\partial\left(\rho_{c} \omega\right)}{\partial x_{j}}=\frac{\partial}{\partial x_{j}}\left[\frac{1}{\operatorname{Pr}_{\omega}} \frac{K}{\omega} \frac{\partial\left(\rho_{c} \omega\right)}{\partial x_{j}}\right]+C_{\omega 1} \rho_{c} \operatorname{prod} \frac{\omega}{K}-C_{\omega 2} \rho_{c} \omega^{2}+S_{\omega}$

For simplicity the overbar indicating turbulence averaging has been dropped in the above equa-

tions, except on the correlations

disperse phase [4,7-9]. We have presented the final mass and momentum equations for both phases in Table 1 (see Eqs. T3-T6). The terms on Eqs. T4, T5 and T6 have the same meaning as explained above. In Eqs. T3 to T6, the subscript $m$ stands for the mixture, and $\rho_{0}$ is a reference density value. For the mixture shear stresses, we assumed $T_{i j, m}^{R e}=-\rho_{m} \overline{u_{i, m}^{\prime} u_{j, m}^{\prime}}$. The 


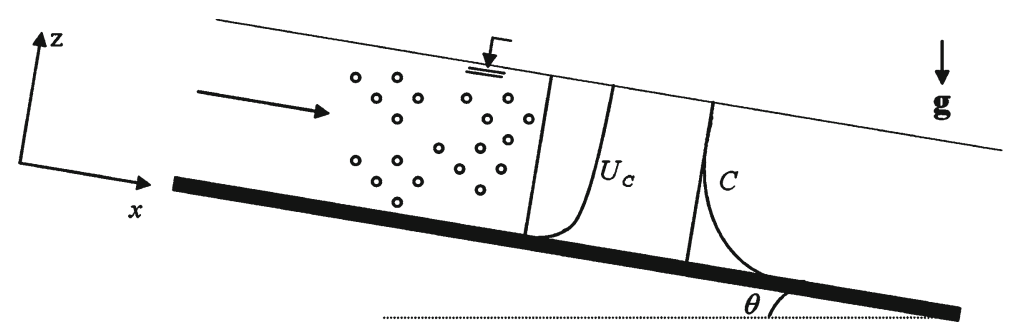

Fig. 1 Schematic of sediment-laden, open-channel flow

PTFM can be simplified further by assuming an algebraic momentum equation for the vertical component of the velocity, in terms of the fall (settling) velocity $\left(W_{s}\right)$ as detailed in [6].

The third and most common approach to model sediment transport is referred to herein as the SSTM [6], which also assumes the mixture to be a surrogate of the carrier fluid; thus, the transport equations for the mixture variables can be expressed through Eqs. T3 and T4. In order to calculate the distribution of sediment, a straightforward advection-diffusion equation, presented in Eq. T7, is solved. In Eq. T7, $W_{s}$ represents the fall velocity which is a positive value, and $C$ indicates the sediment concentration; $\delta_{i j}$ is the Kronecker delta. It is worth mentioning that both $\alpha_{d}$ and the volumetric concentration $C$ have the same physical meaning.

In [6], we presented 1D versions of the above equations. In the case of a 1D sedimentladen, open-channel flow over a plane bed of small slope (shown in Fig. 1), the derivatives in $x$ and $y$ can be disregarded. (The $x, y$, and $z$ axes refer to the stream-wise, transverse and wall-normal directions, respectively.)

\subsection{Closure of the equations: turbulence models for single-phase flows}

The equations presented in Table 1 are not closed, since adequate expressions for the following terms are needed: $\overline{u_{i, c}^{\prime} u_{j, c}^{\prime}}, \overline{u_{i, d}^{\prime} u_{j, d}^{\prime}}, \overline{\alpha_{d}^{\prime} u_{j, d}^{\prime}}$, and $\overline{c^{\prime} u_{j, d}^{\prime}}$. In the case of dilute flows, the stresses of the disperse phase $\overline{u_{i, d}^{\prime} u_{j, d}^{\prime}}$ can be neglected [32]. This is in agreement with results obtained by Bombardelli and Jha [6], who verified that the numerical results with a null value of the eddy viscosity of the disperse phase were closer to the observed data than predictions using the relation proposed by Chen and Wood [14], when the $K-\varepsilon$ model was used. In turn, the Reynolds stresses in the carrier phase $\overline{u_{i, c}^{\prime} u_{j, c}^{\prime}}$ can be obtained "rigorously" by solving the transport equations of the RSM, which in their single-phase version [57] are presented in Eq. T8. In Eq. T8, $P_{i j}$ is the rate of turbulence production by shear; $D_{i j}$ is the diffusion tensor; $\pi_{i j}$ is the pressure-rate-of-strain term; and $\varepsilon_{i j}$ is the rate of dissipation of TKE tensor, defined in Eqs. T9 and T10.

The pressure-rate-of-strain tensor above was modeled using the LRR-IP model [40]. In the Eqs. T9 and T10, prod is the turbulent shear production ( $p r o d=-\overline{u_{i}^{\prime} u_{j}^{\prime}} \partial U_{i} / \partial x_{j}$ ), and $C_{s}, C_{1}, C_{2}$ are constants. The TKE in the equations can be obtained by adding the diagonal terms of the Reynolds stress tensor or, alternatively, by solving the transport equations of the $K-\varepsilon$ model $[6,58]$. We adopted the latter approach herein. The solid fraction Reynolds fluxes, i.e., $\overline{\alpha_{d}^{\prime} u_{j, d}^{\prime}}$ and $\overline{c^{\prime} u_{j, d}^{\prime}}$, can be computed using the definition of the Schmidt number $(\sigma)$ following Eq. T13; in turn, the $K-\varepsilon$ model was employed to provide the eddy viscosity. ( $\sigma$ is defined as the ratio between the eddy viscosity of the carrier phase and the diffusivity of suspended sediment.) In this paper, we propose an extension of the RSM to address two-phase flows in Sect. 4. 
The ASM has the advantage over the RSM that it is easier to implement. The standard approach in deriving the ASM is based on the assumption that the convective and diffusive transport terms of individual Reynolds stress components are proportional to the transport of TKE [58]. The final expression for the stresses is presented in Eq. T11 in Table 1. In Eq. T11 $C_{1}$ and $C_{2}$ are the same constants of the RSM.

The $K-\varepsilon$ and the $K-\omega$ models are based on the Boussinesq model $[57,58]$. The Reynolds stresses and Reynolds fluxes are expressed in terms of the gradients of the time-averaged velocities and concentrations, as depicted in Eqs. T12 and T13, respectively. In Eqs. T12 and $\mathrm{T} 13, v_{T}$ is the eddy viscosity of the carrier, computed as $v_{T}=\frac{\mu_{T}}{\rho}=C_{\mu} \frac{K^{2}}{\varepsilon}=\frac{K}{\omega}$ in the case of the $K-\varepsilon$ and the $K-\omega$ models, respectively, with $\mu_{T}$ indicating the dynamic eddy viscosity of the carrier. The transport equation for $K$ (Eq. T14) is the same in both the $K-\varepsilon$ and $K-\omega$ models (obviously changing the expression for $\varepsilon$ as a function of $\omega: \varepsilon=C_{\mu} K \omega$ ). Equations T15 and T16 are the transport equations for the DTKE, $\varepsilon$, and the specific dissipation, $\omega$.

\section{Extended versions of the RSM and the $K-\varepsilon$ and $K-\omega$ models for two-phase flows}

\subsection{Extension of the RSM}

The derivation of the transport equations for the stresses $\overline{u_{i, c}^{\prime} u_{j, c}^{\prime}}$ of the carrier phase contains the following steps:

(a) Multiply the momentum equation for the fluctuation velocity $\left(u_{i}^{\prime}\right)$ by $u_{j}^{\prime}$;

(b) interchange subscripts $i$ and $j$;

(c) add equations obtained in steps (a) and (b) and average;

(d) apply the product rule.

The details of those mathematical derivations are presented in the Appendix. The last two terms of Eq. A.12 arise as a consequence of having interaction forces between phases. Further considerations are needed in order to close those additional terms, which are provided on the Appendix, leading to the following final expression for the stresses:

$$
\frac{D}{D t}\left[\alpha_{c} \rho_{c}\left(\overline{u_{i, c}^{\prime} u_{j, c}^{\prime}}\right)\right]=P_{i j}+D_{i j}+\pi_{i j}-\varepsilon_{i j}+S_{u_{i}^{\prime} u_{j}^{\prime}}
$$

with

$$
S_{u_{i}^{\prime} u_{j}^{\prime}}=-2 \beta\left(1-f_{u}\right)\left(\overline{u_{i, c}^{\prime} u_{j, c}^{\prime}}\right)+\frac{3}{4 d_{p}} C_{D}\left|\overrightarrow{U_{r}}\right|\left[U_{i, r} D_{d} \frac{\partial \alpha_{d}}{\partial x_{j}}+U_{j, r} D_{d} \frac{\partial \alpha_{d}}{\partial x_{i}}\right]
$$

Since our models focus on dilute conditions and since the selected datasets pertain to such conditions (see Sect.5), we did not include stratification effects in the model extensions.

\subsection{Extension of the $K-\varepsilon$ model}

Herein, we employed an extended version of the $K-\varepsilon$ model similar to that of [32]. Some authors have also used volume fractions of the carrier phase multiplying terms in the equations for the TKE and the DTKE. We believe that this is not required, given the dilute-mixture nature of the flows analyzed in this paper. Hsu et al. proposed the following expressions for $S_{K}$ and $S_{\varepsilon}$, the terms they added to the $K-\varepsilon$ model to account for the presence of a second phase (see Eqs. T14 and T15): 
with

$$
S_{K}=S_{K 1}+S_{K 2} ; \quad S_{\varepsilon}=1.2 S_{K} \frac{\varepsilon}{K}
$$

$$
\begin{aligned}
& S_{K 1}=-\frac{2 \rho_{d} \alpha_{d} K}{T_{P}+T_{L}}, \text { and } \\
& S_{K 2}=\frac{3}{4 d_{p}} \rho_{c} C_{D}\left|\overrightarrow{U_{r}}\right| \nu_{T} \frac{\partial \alpha_{d}}{\partial x_{i}}\left(U_{i, c}-U_{i, d}\right)
\end{aligned}
$$

In the above equations, $S_{K 1}$ represents the correlation between the fluid and sediment velocity fluctuations and $S_{K 2}$ represents the production of TKE due to the drag force [32,39]. Notice that $S_{K}$, and $S_{\varepsilon}$ become zero when $\alpha_{d}=0$, as expected. In turn, the scales $T_{P}$ (particle time scale) and $T_{L}$ (flow time scale) are given by:

$$
T_{P}=\frac{\rho_{d}}{\frac{3}{4 d_{p}} \rho_{c} C_{D}\left|\vec{U}_{r}\right|} ; \quad T_{L}=0.165 \frac{K}{\varepsilon}
$$

We adapted this particle time scale from Hsu et al.'s expression for a non-linear drag force.

\subsection{Extension of the $K-\omega$ model}

We followed similar procedures to those of [32] to extend the $K-\varepsilon$ model. To the best of our knowledge, no extension of this model for two-phase flows has been provided before (besides the attempt by Yoon and Kang [74] mentioned above). The final equation for $\omega$ is the same as Eq. T16, in which

$$
\begin{gathered}
S_{\omega}=1.2 S_{K} \frac{\omega}{K} \\
S_{K}=-\frac{2 \rho_{d} \alpha_{d} K}{T_{P}+T_{L}}+\frac{3}{4 d_{p}} \rho_{c} C_{D}\left|\vec{U}_{r}\right| \nu_{T} \frac{\partial \alpha_{d}}{\partial x_{i}}\left(U_{i, c}-U_{i, d}\right)
\end{gathered}
$$

In Table 2, we present the governing equations of the different models and the diverse turbulence closures in their 1D forms for the open-channel problem. The values of the constants are also listed in Table 2.

\subsection{Considerations about the values of the model constants}

It is worth pointing out here that the values of the constants are the same as those developed for single-phase flows. Ideally, those constants should be modified in order to account for the presence of a particulate phase in sediment-laden flows. To determine those values would require an appropriate and detailed experimental dataset (non-existent to date to the best of our knowledge) or Direct Numerical Simulation (DNS) results, as done by Squires and Eaton [64]. For the constants involved in the $K-\varepsilon$ model, Squires and Eaton [64] concluded that for dilute flows with mass loadings below 0.2 , the modification in the values of the constants are small (although some caution should be taken in cases of preferential concentration of particles). Therefore, we have used the unmodified values of the constants in the turbulence models, following customary approaches in most papers (see, for instance, $[2,14,23,32,55,73])$.

In the case of the $K-\omega$ model, $[43,74]$ have used the same constants that were suggested by Wilcox [72]. Similarly, in the case of the ASM and the RSM, we found that researchers have used the same constants for the turbulence models as they were developed for clear water flows (e.g., $[1,15,60])$. 
Table 2 Governing equations used in different modeling approaches in 1D

\begin{tabular}{|c|c|c|}
\hline Models & & Governing equations \\
\hline \multirow[t]{8}{*}{ SSTM } & Carrier phase & \\
\hline & Mass balance & $\frac{\partial W_{m}}{\partial z}=0$ \\
\hline & Momentum balance in $\mathrm{x}$ & $\frac{\partial\left(\rho_{m} U_{m}\right)}{\partial t}=-\frac{\partial}{\partial z}\left[\rho_{m} \overline{u_{m}^{\prime} w_{m}^{\prime}}\right]+\rho_{m} g S$ \\
\hline & Momentum balance in $\mathrm{z}$ & $W_{m}=0$ \\
\hline & Disperse phase & \\
\hline & Mass balance & $\frac{\partial C}{\partial t}+W_{d} \frac{\partial C}{\partial z}=-\frac{\partial}{\partial z}\left[\overline{w_{d}^{\prime} c^{\prime}}\right]$ \\
\hline & Momentum balance in $\mathrm{x}$ & $U_{d}=U_{m}$ \\
\hline & Momentum balance in $\mathrm{z}$ & $W_{d}=-W_{s}$ \\
\hline \multirow[t]{8}{*}{ PTFM } & Carrier phase & \\
\hline & Mass balance & $\frac{\partial W_{m}}{\partial z}=0$ \\
\hline & Momentum balance in $\mathrm{x}$ & $\frac{\partial\left(\rho_{m} U_{m}\right)}{\partial t}=-\frac{\partial}{\partial z}\left[\rho_{m} \overline{u_{m}^{\prime} w_{m}^{\prime}}\right]+\rho_{m} g S$ \\
\hline & Momentum balance in $\mathrm{z}$ & $W_{m}=0$ \\
\hline & Disperse phase & \\
\hline & Mass balance & $\frac{\partial\left(\alpha_{d} \rho_{d}\right)}{\partial t}+\frac{\partial\left(\alpha_{d} \rho_{d} W_{d}\right)}{\partial z}=-\frac{\partial}{\partial z}\left[\rho_{d} \overline{w_{d}^{\prime} \alpha_{d}^{\prime}}\right]$ \\
\hline & Momentum balance in $\mathrm{x}$ & $\begin{array}{l}\frac{\partial\left(\alpha_{d} \rho_{d} U_{d}\right)}{\partial t}+\frac{\partial\left(\alpha_{d} \rho_{d} U_{d} W_{d}\right)}{\partial z}=-\frac{\partial}{\partial z}\left[\rho_{d} \alpha_{d} \overline{u_{d}^{\prime} w_{d}^{\prime}}\right. \\
\quad+\alpha_{d} \rho_{d} g S+F_{D, x}\left(^{*}\right)\end{array}$ \\
\hline & Momentum balance in $\mathrm{z}$ & $W_{d}=-W_{s}$ \\
\hline \multirow[t]{9}{*}{ CTFM } & Carrier phase & \\
\hline & Mass balance & $\frac{\partial W_{C}}{\partial z}=0$ \\
\hline & Momentum balance in $\mathrm{x}$ & $\begin{array}{l}\frac{\partial\left[\left(1-\alpha_{d}\right) \rho_{c} U_{c}\right]}{\partial t}=-\frac{\partial}{\partial z}\left[\left(1-\alpha_{d}\right) \rho_{c} \overline{u_{c}^{\prime} w_{c}^{\prime}}\right] \\
\quad+\left(1-\alpha_{d}\right) \rho_{c} g S-F_{D, x}\end{array}$ \\
\hline & Momentum balance in $\mathrm{z}$ & $W_{c}=0$ \\
\hline & Disperse phase & \\
\hline & Mass balance & $\frac{\partial\left(\alpha_{d} \rho_{d}\right)}{\partial t}+\frac{\partial\left(\alpha_{d} \rho_{d} W_{d}\right)}{\partial z}=-\frac{\partial}{\partial z}\left[\rho_{d} \overline{w_{d}^{\prime} \alpha_{d}^{\prime}}\right]$ \\
\hline & Momentum balance in $\mathrm{x}$ & $\frac{\partial\left(\alpha_{d} \rho_{d} U_{d}\right)}{\partial t}+\frac{\partial\left(\alpha_{d} \rho_{d} U_{d} W_{d}\right)}{\partial z}=-\frac{\partial}{\partial z}\left[\alpha_{d} \rho_{d} \overline{u_{d}^{\prime} w_{d}^{\prime}}\right.$ \\
\hline & & $+\alpha_{d} \rho_{d} g S+F_{D, x}(* *)$ \\
\hline & Momentum balance in $\mathrm{z}$ & $\begin{array}{l}\frac{\partial\left(\alpha_{d} \rho_{d} W_{d}\right)}{\partial t}+\frac{\partial\left(\alpha_{d} \rho_{d} W_{d} W_{d}\right)}{\partial z}=-\alpha_{d} \frac{\partial P_{c}}{\partial z} \\
\quad-\frac{\partial}{\partial z}\left[\alpha_{d} \rho_{d} \overline{w_{d}^{\prime} w_{d}^{\prime}}\right]-\alpha_{d} \rho_{d} g \cos \theta+F_{D, z}(* *)\end{array}$ \\
\hline \multirow[t]{5}{*}{$K-\varepsilon$ model } & & $\begin{array}{l}-\overline{-u_{m}^{\prime} w_{m}^{\prime}}=v_{T} \frac{\partial U_{m}}{\partial z} ; \overline{-u_{c}^{\prime} w_{c}^{\prime}}=v_{T} \frac{\partial U_{c}}{\partial z} \\
\text { where, } v_{T}=\frac{\mu_{T}}{\rho}=C_{\mu} \frac{K^{2}}{\varepsilon}\end{array}$ \\
\hline & Turbulent kinetic energy (TKE) & $\frac{\partial K}{\partial t}=\frac{\partial}{\partial z}\left[\frac{C_{\mu}}{\sigma_{k}} \frac{K^{2}}{\varepsilon} \frac{\partial K}{\partial z}\right]+v_{T}\left(\frac{\partial U_{c}}{\partial z}\right)^{2}-\varepsilon+\xi S_{K}$ \\
\hline & $\begin{array}{l}\text { Dissipation rate of } \\
\text { turbulent kinetic } \\
\text { energy (DTKE) }\end{array}$ & $\begin{array}{l}\frac{\partial \varepsilon}{\partial t}=\frac{\partial}{\partial z}\left[\frac{C_{\mu}}{\sigma_{\varepsilon}} \frac{K^{2}}{\varepsilon} \frac{\partial \varepsilon}{\partial z}\right]+C_{\varepsilon 1} \nu_{T}\left(\frac{\partial U_{c}}{\partial z}\right)^{2} \frac{\varepsilon}{K}- \\
C_{\varepsilon 2} \frac{\varepsilon^{2}}{K}+\xi S_{\varepsilon}\end{array}$ \\
\hline & & where, $S_{K}=S_{K 1}+S_{K 2} ; \quad S_{\varepsilon}=1.2 S_{K} \frac{\varepsilon}{K}$ \\
\hline & & $\begin{array}{l}S_{K 1}=-\frac{2 \rho_{d} \alpha_{d} K}{T_{P}+T_{L}} ; \text { and } S_{K 2}=\frac{3}{4 d_{p}} \rho_{c} C_{D}\left|\overrightarrow{U_{r}}\right| \\
\quad v_{T} \frac{\partial \alpha_{d}}{\partial z}\left(W_{c}-W_{d}\right)\end{array}$ \\
\hline
\end{tabular}


Table 2 continued

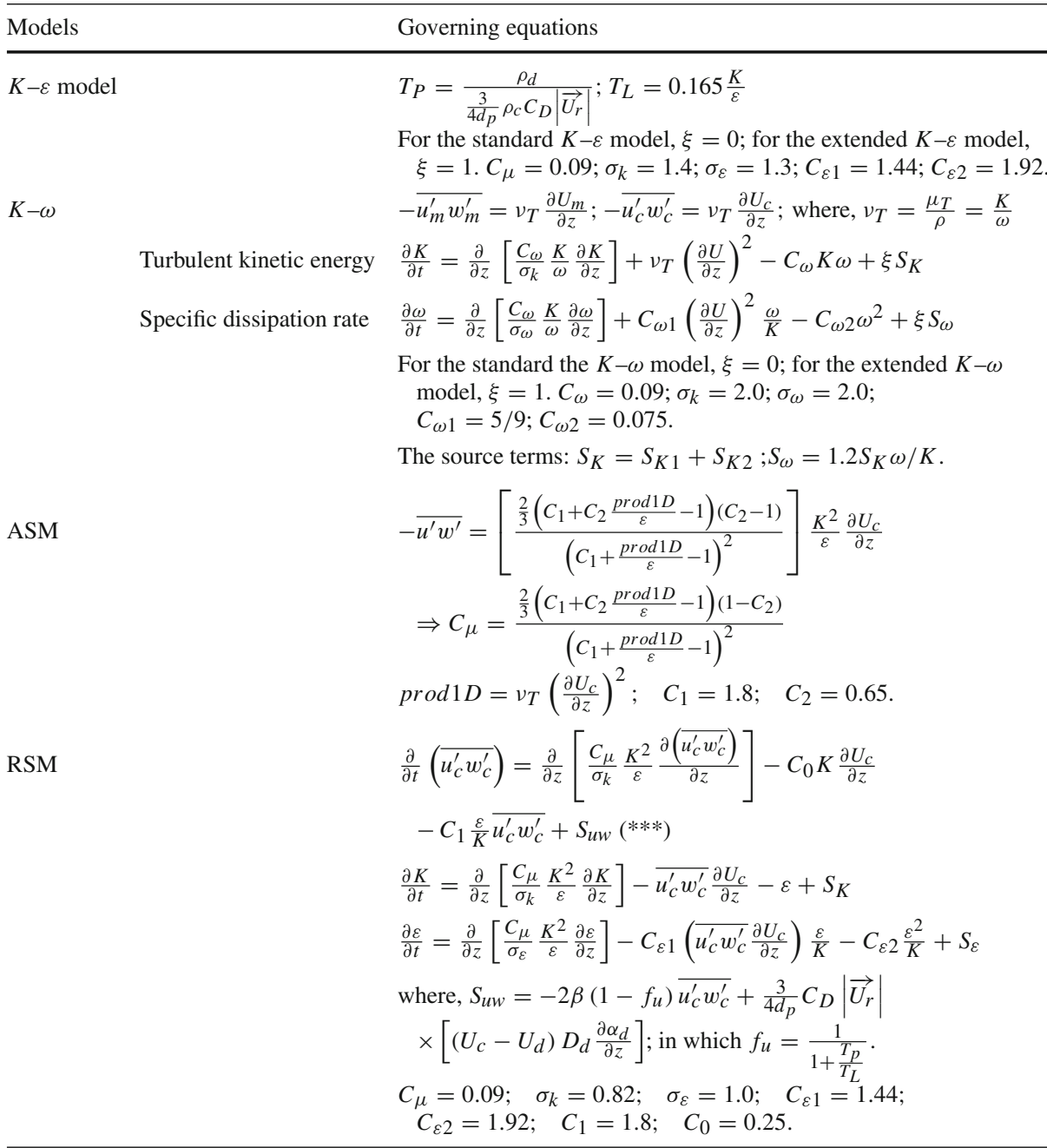

Turbulence closures expressed in 1D

* The diffusive term in the equation of momentum in $x$ of the disperse phase needs to be considered as a model in itself, since triple correlations such as $\left(\overline{u_{d}^{\prime} w_{d}^{\prime} \alpha_{d}^{\prime}}\right)$ have been disregarded

** $F_{D, x}$ and $F_{D, z}$ were modeled following [6]

*** The diffusion of Reynolds stresses and terms involving normal stresses have been approximated as a function of the TKE by following Lien and Leschziner (1994)

\section{Runs with different model variants and boundary conditions}

\subsection{Runs developed}

We performed diverse runs listed in Table 3, by combining seven different turbulence closures (i.e., the standard and extended $K-\varepsilon$ model, the standard and extended $K-\omega$ model, the ASM and the standard and extended RSM) with the theoretical framework composed by the 
Table 3 List of runs performed

\begin{tabular}{lll}
\hline Run & Model & Turbulence model \\
\hline 1 & SSTM & Standard $K-\varepsilon$ \\
2 & SSTM & Standard $K-\omega$ \\
3 & SSTM & Standard ASM \\
4 & SSTM & Standard RSM \\
5 & PTFM & Standard $K-\varepsilon$ \\
6 & PTFM & Standard $K-\omega$ \\
7 & PTFM & Standard ASM \\
8 & PTFM & Standard RSM \\
9 & PTFM & Extended $K-\varepsilon$ \\
10 & PTFM & Extended $K-\omega$ \\
11 & PTFM & Extended RSM \\
12 & CTFM & Standard $K-\varepsilon$ \\
13 & CTFM & Standard $K-\omega$ \\
14 & CTFM & Standard ASM \\
15 & CTFM & Standard RSM \\
16 & CTFM & Extended $K-\varepsilon$ \\
17 & CTFM & Extended $K-\omega$ \\
18 & CTFM & Extended RSM \\
\hline
\end{tabular}

Table 4 Summary of flow characteristics in the experiments selected ${ }^{\mathrm{a}}$

\begin{tabular}{llllllllr}
\hline $\begin{array}{l}\text { Reference } \\
\text { data source }\end{array}$ & Case & Depth $(\mathrm{cm})$ & $\begin{array}{l}\text { Slope } \\
\left(\times 10^{-3}\right)\end{array}$ & $\begin{array}{l}\text { Particle } \\
\text { diameter } \\
(\mathrm{mm})\end{array}$ & $\begin{array}{l}\text { Settling (fall) } \\
\text { velocity }(\mathrm{m} / \mathrm{s})\end{array}$ & $\begin{array}{l}\text { Shear } \\
\text { velocity } \\
(\mathrm{m} / \mathrm{s})\end{array}$ & $\begin{array}{l}\rho_{d} \\
\left(\mathrm{~kg} / \mathrm{m}^{3}\right)\end{array}$ & $R e_{p}$ \\
\hline$[69]$ & 18 & 14.08 & 1.25 & 0.134 & 0.00838 & 0.041 & 2,650 & 6.24 \\
& 19 & 7.19 & 1.25 & 0.1 & 0.00829 & 0.041 & 2,650 & 4.02 \\
{$[19]$} & 23 & 17.0 & 2.0 & 0.210 & 0.023 & 0.041 & 2,650 & 12.24 \\
& 26 & 17.1 & 2.0 & 0.210 & 0.023 & 0.041 & 2,650 & 12.24 \\
& 29 & 16.8 & 2.0 & 0.210 & 0.023 & 0.041 & 2,650 & 12.24 \\
{$[50]$} & SL01 & 12.9 & 0.739 & 0.25 & 0.024 & 0.0324 & 2,650 & 15.90 \\
{$[53]$} & PS05 & 5.0 & 1.2 & 0.5 & 0.0068 & 0.0147 & 1,050 & 7.83 \\
{$[51]$} & NS1 & 2.1 & 11.3 & 0.25 & 0.024 & 0.042 & 2,650 & 15.90 \\
\hline
\end{tabular}

The settling velocity in these tests was obtained from formulas; it was not measured. $R e_{p}$ is given by $\sqrt{\left(g d_{p}^{3} \Delta \rho / \rho\right)} / v$; where $v$ is the kinematic viscosity of the carrier phase

a All tests pertain to dilute flow conditions

SSTM, the PTFM and the CTFM. Note that there is no need to employ extended versions of the models with the SSTM because the SSTM does not incorporate interaction forces in any of the momentum equations. Numerical results were compared with the observations of [19,50,53,69 and 51]; details of the experiments are shown herein in Table 4. Runs were performed with the same number of volumes as determined by the mesh-convergence analysis of [6]. We also refer to that paper for more information on the numerical treatment of the equations. Runs were performed for $10,000 \mathrm{~s}$ of simulation time, using the time step as a relaxation to the steady state. The steady state was achieved after $1,000 \mathrm{~s}$ in most runs (see [6]). 


\subsection{Boundary conditions}

We employed mostly the same boundary conditions discussed in [6], with the natural additions of those boundary conditions for the other turbulence models developed and used in this work. Similar to [6], the value of $\alpha_{d}$ at the first computational point $\left(\alpha_{d, b}\right)$ was adopted to be the largest measured value close to the bed. We also present herein an assessment of the boundary condition for the sediment volume fraction at the free surface in Sect. 6.5. For the $K-\omega$ model, we used the same boundary conditions employed by Wilcox [72] and López and García [43], which read:

$$
\left.\omega\right|_{z=0}=U_{*} /\left(0.3 \kappa y_{1}\right) ;\left.\quad(\partial \omega / \partial z)\right|_{z=h}=0
$$

In the above equations $y_{1}$ is a convenient distance from the wall; $U_{*}$ is the wall-friction (shear) velocity; and $\kappa$ indicates the von Kármán constant. Please recall that the condition $\left.\omega\right|_{z=0}$ is valid for a point outside the viscous sublayer.

In our previous work [6], we observed that the value of the non-dimensional shear stress $-\left.\overline{u^{\prime} w^{\prime}}\right|_{z=0} / U_{*}^{2}$, computed using the $K-\varepsilon$ model, was close to 0.5 at the bottom, for all tests. Therefore, we applied that same boundary condition on the shear stress at the bottom in these runs (for the RSM and the ASM). At the top boundary, we imposed a null value for the shear stress: $\left.\overline{u^{\prime} w^{\prime}}\right|_{z=h}=0$.

\section{Comparison of simulation results with experiments and discussion}

\subsection{Mean velocity of the carrier phase}

Figures 2, 3, 4, 5 and 6 depict the measured and modeled profiles of the mean velocity of the carrier phase in the wall-normal direction. In Figs. 2, 3 and 4, we compare the performance of the turbulence closures in their standard versions with data for each member of the modeling framework (the SSTM, the PTFM and the CTFM, respectively). We note that for the test case of Muste et al., the numerical results using the standard $K-\varepsilon$ model show under-prediction of the data points in the region $0<z / h<0.3$, while they show a slight over-prediction in the upper region $0.3<z / h<1$, for all approaches within the framework. The results with the $K-\omega$ model show a small under-prediction throughout the entire depth when using the SSTM and the PTFM, and a slightly better prediction with the CTFM. When we use the ASM, the results are close to those obtained using the $K-\varepsilon$ model in the lower $20 \%$ of the depth, but overall the ASM performs better than the $K-\varepsilon$ model. Results with the RSM show the best agreement with data for the observations by Muste et al. Largest differences among predictions and data occur in the lower $5 \%$ of the depth, but differences are between 5 and $10 \%$.

For the test case of Muste and Patel, all models seem to follow a common bundle up to mid depth, deviating afterwards. The $K-\varepsilon$ turbulence closure gives better predictions than other closures for larger relative distances from the wall. Despite these relative considerations, we observe that, overall, the predictions using different turbulence closures in their standard, single-phase versions are within $5-10 \%$ from each other. This result is valid for both datasets.

In Figs. 5 and 6, we present the comparison of numerical results with data when the RSM and the $K-\omega$ model are used in their standard as well as in their extended forms. The results 


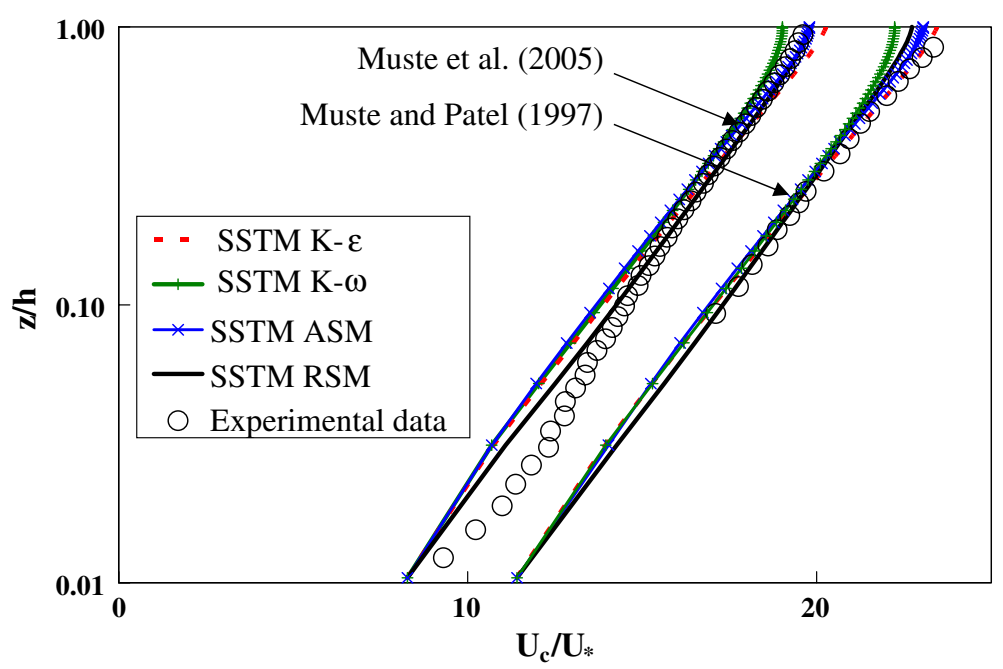

Fig. 2 Comparison of simulated values of the stream-wise velocity of the carrier phase with the experimental data. Simulations were performed using the standard $K-\varepsilon$ and $K-\omega$ models, the ASM and the RSM, under the approach of the SSTM

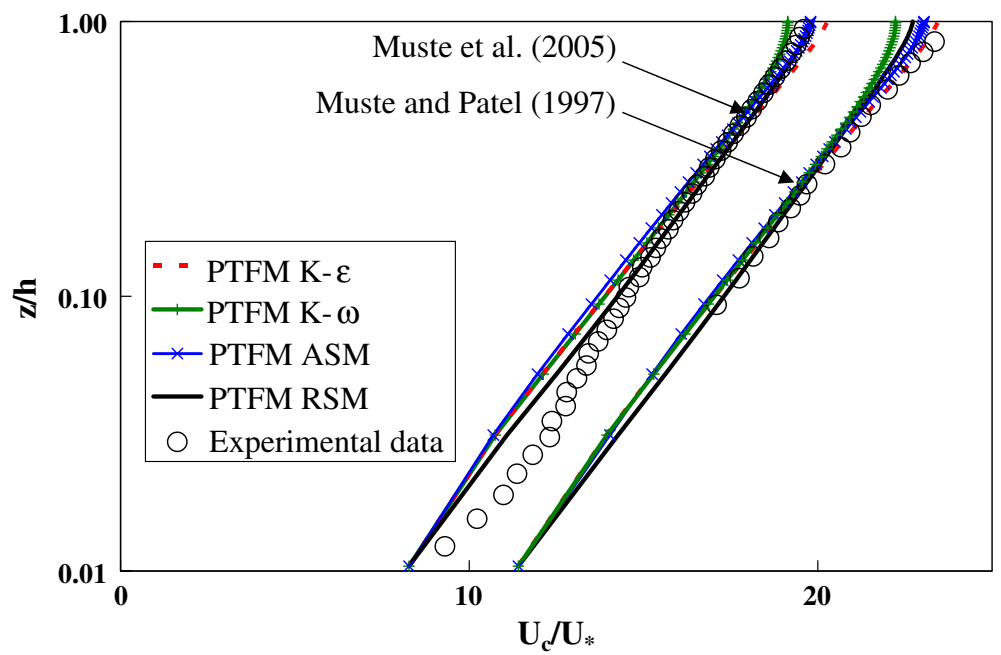

Fig. 3 Comparison of simulated values of the stream-wise velocity of the carrier phase with the experimental data. Simulations were performed using the standard $K-\varepsilon$ and $K-\omega$ models, the ASM and the RSM, under the approach of the PTFM

show that the additional terms in the RSM and the $K-\omega$ model only affect slightly the model predictions ( $5 \%$ of difference at most). Differences are also small for the $K-\varepsilon$ model, as shown by Bombardelli and Jha [6]. Interestingly, while the extended RSM produces a less-accurate prediction in the test case of Muste et al., not much change is observed in the test case of Muste and Patel. The additional terms in the $K-\omega$ model produce an improvement for the test case of Muste et al. This result is as equally valid for the PTFM (Fig. 5) as it is for the CTFM (Fig. 6). 


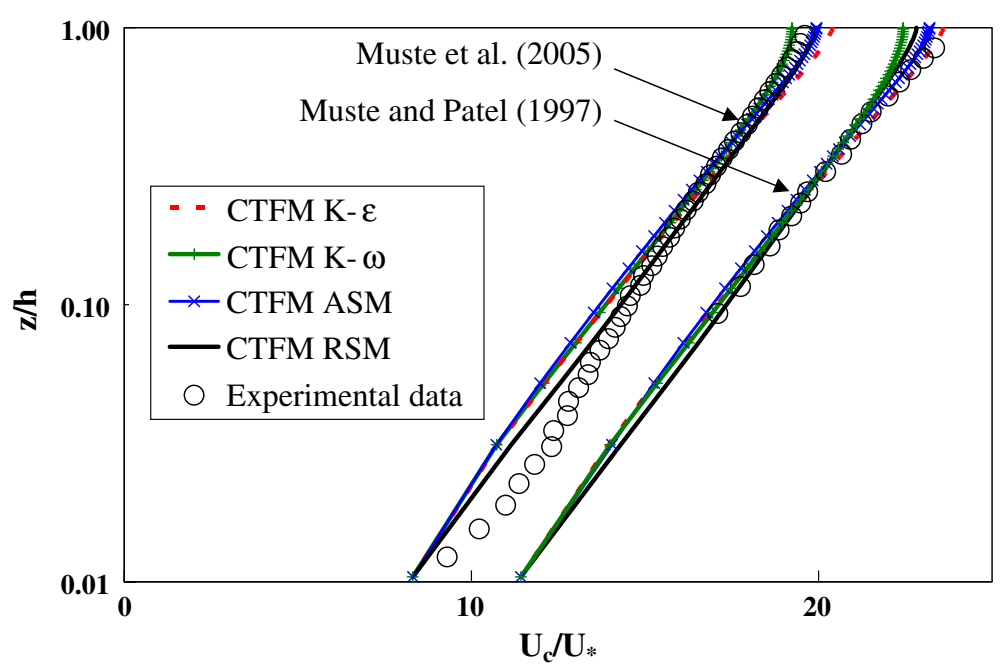

Fig. 4 Comparison of simulated values of the stream-wise velocity of the carrier phase with the experimental data. Simulations were performed using the standard $K-\varepsilon$ and $K-\omega$ models, the ASM and the RSM, under the approach of the CTFM

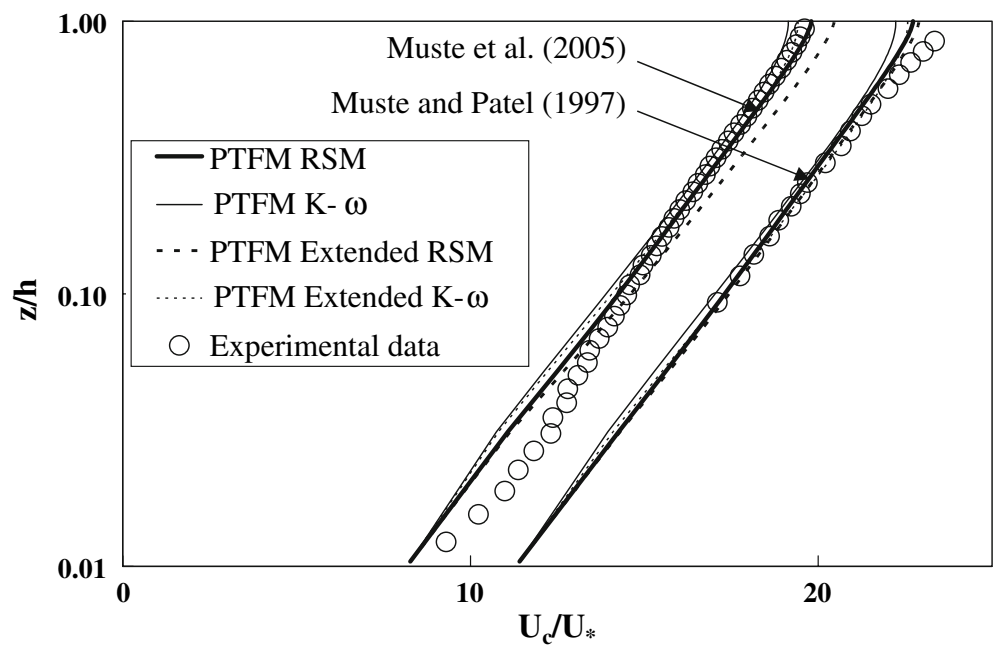

Fig. 5 Comparison of simulated values of the stream-wise velocity of the carrier phase with the experimental data. Simulations were performed using the RSM and the $K-\omega$ model in their standard as well as extended forms, under the approach of the PTFM

\subsection{Mean velocity of the disperse phase}

The numerical results in terms of the mean stream-wise velocity of the disperse phase, for the PTFM and the CTFM are presented in Figs. 7 and 8. It becomes apparent that predictions using very different turbulence closures almost overlap with each other in more than half the depth for the test case of Muste and Patel, and are very close in the test case of Muste et al. In the case of the PTFM, the ASM seems to provide more accurate predictions for the case 


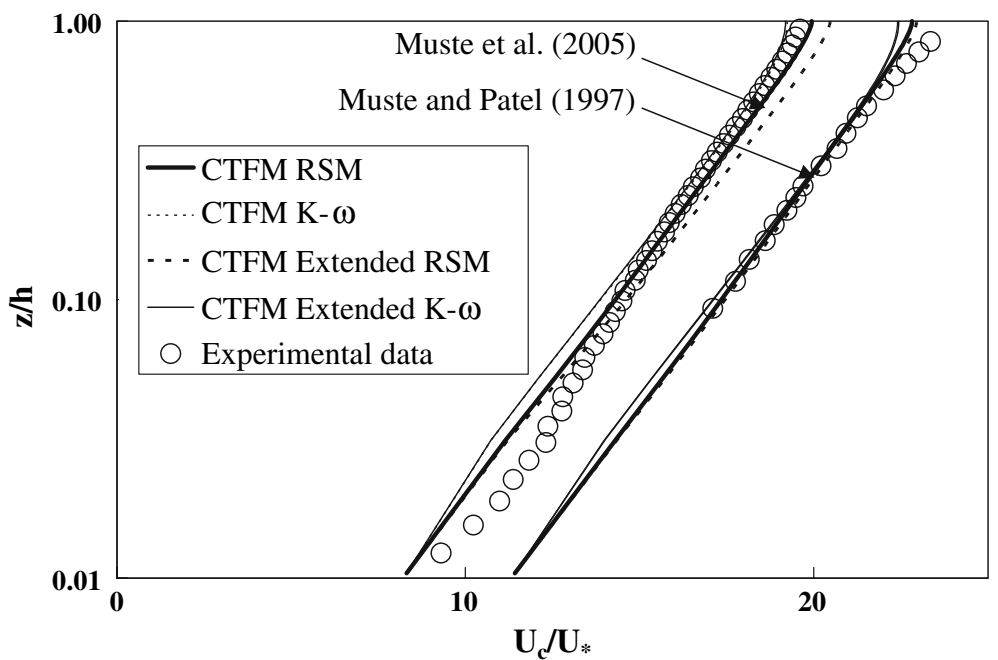

Fig. 6 Comparison of simulated values of the stream-wise velocity of the carrier phase with the experimental data. Simulations were performed using the RSM and the $K-\omega$ model in their standard as well as extended forms, under the approach of the CTFM

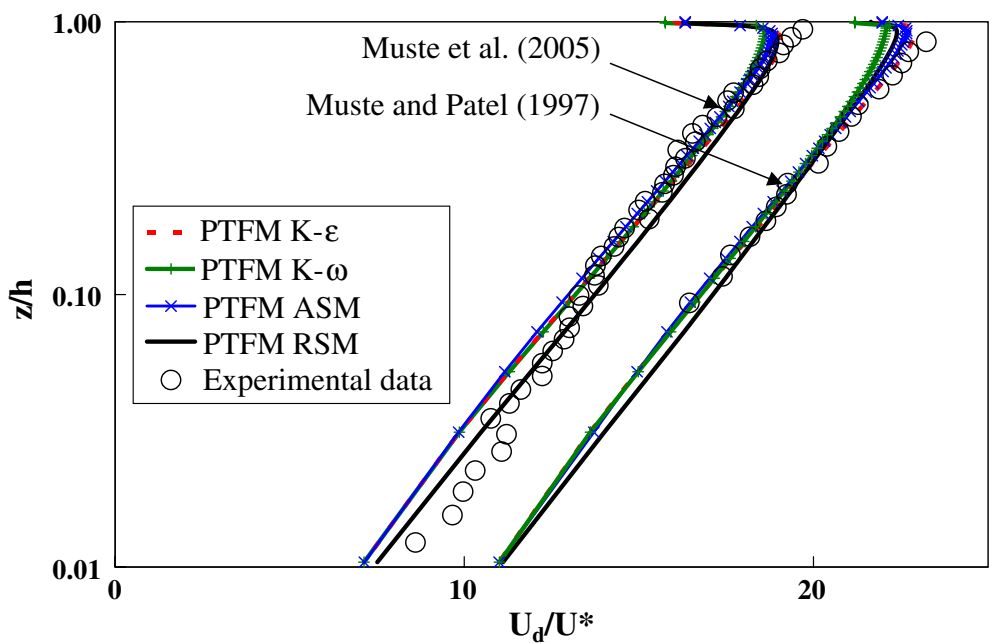

Fig. 7 Comparison of simulated values of the stream-wise velocity of the disperse phase with the experimental data. Simulations were performed using the standard $K-\varepsilon$ and $K-\omega$ models, the ASM and the RSM, under the approach of the PTFM

of Muste et al. than other methods, while the RSM does so for the CTFM. For the case of Muste and Patel, the $K-\varepsilon$ model seems to offer the closest agreement. However, not much difference can be observed among the predictions.

We also evaluated in Fig. 9 the effects of extending the RSM and the $K-\omega$ model on the prediction of the stream-wise velocity of the disperse phase. From this figure, it becomes clear that the addition of terms in the turbulence closure does not lead to better predictions in any test case. 


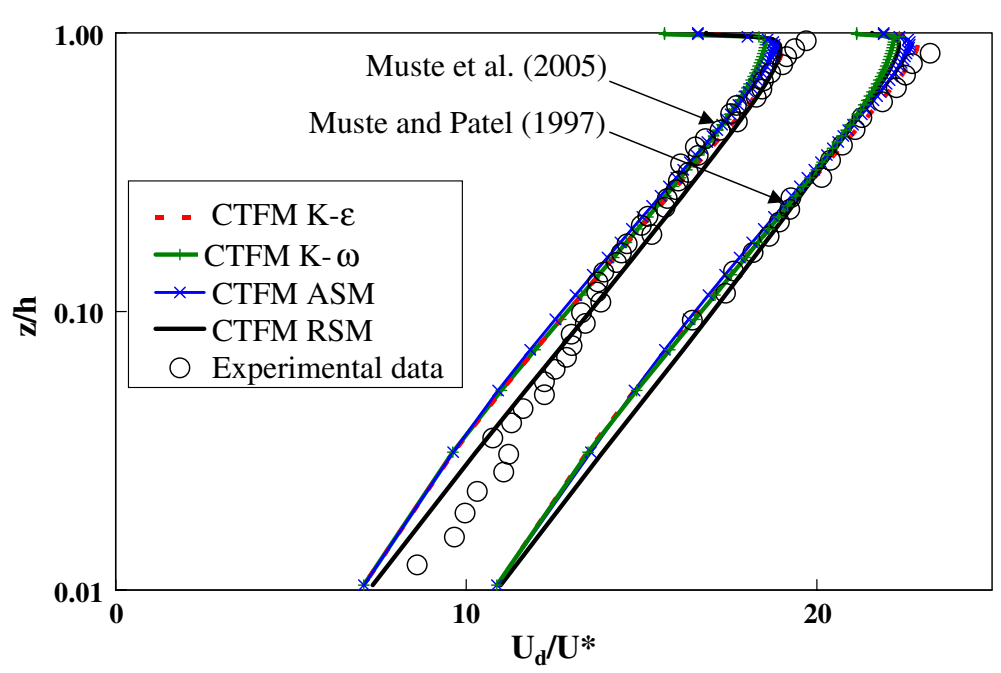

Fig. 8 Comparison of simulated values of the stream-wise velocity of the disperse phase with the experimental data. Simulations were performed using the standard $K-\varepsilon$ and $K-\omega$ models, the ASM and the RSM, under the approach of the CTFM

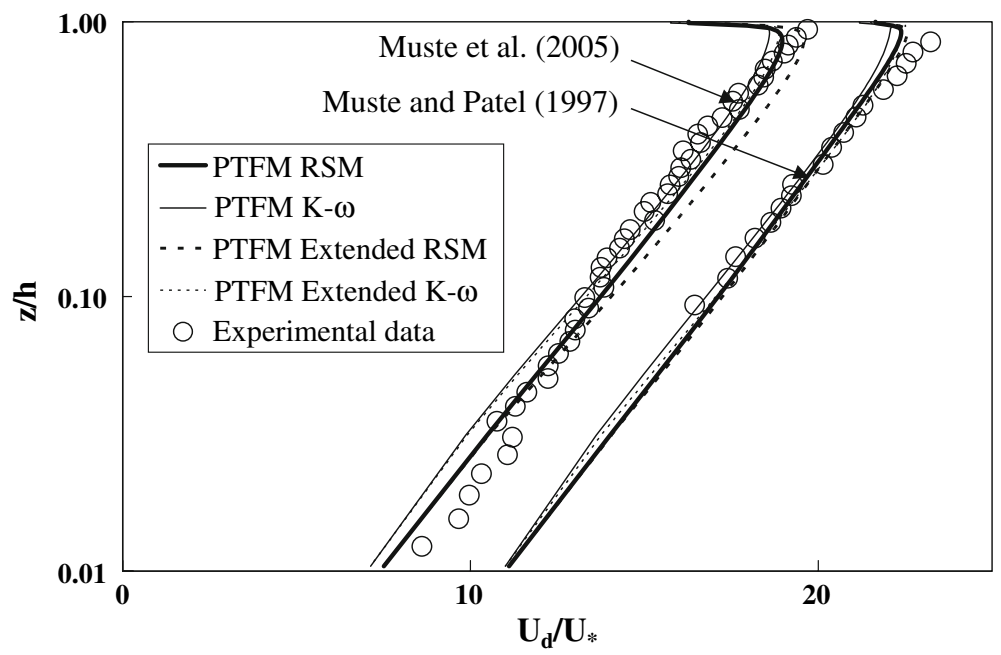

Fig. 9 Comparison of simulated values of the stream-wise velocity of the disperse phase with the experimental data. Simulations were performed using the RSM and the $K-\omega$ model in their standard as well extended forms, under the approach of the PTFM

\subsection{Turbulence statistics}

We present the comparison between simulation results and experimental data of TKE in Fig. 10 for the test case of Muste and Patel. To compute the TKE from the available experimental data, we adopted the same relationship found in our previous paper [6] between the root mean square (rms) of the transverse velocity (axis $y$ ) and the rms of the stream-wise velocity, $\sqrt{\overline{v^{\prime 2}}} \approx 0.3-0.6 \sqrt{\overline{u^{\prime 2}}}$ depending on the dataset. The profiles of TKE using the RSM 


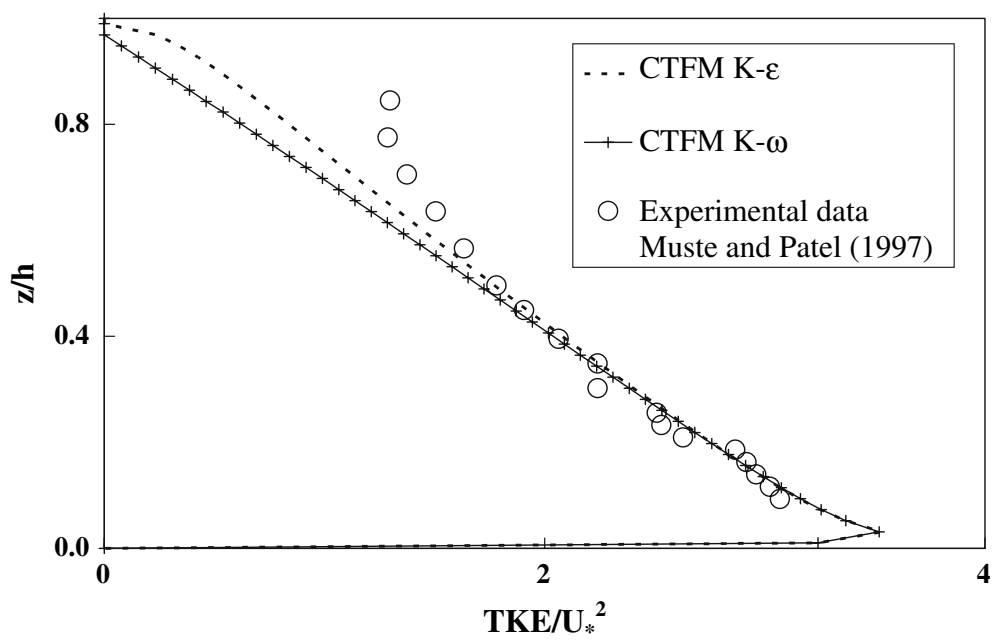

Fig. 10 Comparison of simulated values of the non-dimensional turbulent kinetic energy (TKE) of the carrier phase with the experimental data. Simulations were performed using the $K-\varepsilon$ and $K-\omega$ models under the approach of the CTFM

and the ASM were not included since we did not solve for $\overline{u_{y}^{\prime} u_{y}^{\prime}}$ in our computations. We note that the agreement of model predictions with data is satisfactory up to $\frac{z}{h} \approx 0.75$, but it is not good above that height. This discrepancy can be attributed in part to the boundary condition for $K$ at the free surface: $K=0$. In Sect. 6.5 below we discuss the effects of the boundary condition at the free surface. We observed that the differences in results between the PTFM (not shown herein) and the CTFM (Fig. 10) are not significant. Similar observations can be made for the test case of Muste et al. (not shown herein), which confirms the findings in our previous paper that increasing the complexity of the model does not lead to improvement for dilute sediment transport.

We present the wall-normal distribution of the non-dimensional Reynolds stresses in Fig. 11 for the CTFM (the results for the PTFM are very similar), and the test case of Muste et al. A linear profile of the shear stresses is expected in the case of clear-water flow; a similar profile is also observed here in the case of dilute, sediment-laden flow. We notice that the profiles obtained with different turbulence closures overlap with each other and fall close to the data. However, the RSM deviates from other closures. Unfortunately, the scatter of the data points does not allow for discerning which result is closer to the observations. A very similar pattern was observed for the test case of Muste and Patel, not shown herein.

\subsection{Distribution of the volume fraction of the sediment in the wall-normal direction}

In our previous work [6], we found that the values of the Schmidt number that facilitated the agreement with data, for the case of the PTFM and the $K-\varepsilon$ model, were 0.7 and 0.56 for the tests of [45] and Muste et al., respectively.

In this section, we examine the results for the Schmidt number obtained when the PTFM and the CTFM are both used in combination with different turbulence closures. In Figs. 12 and 13 we present the wall-normal distribution of sediment concentration for the test case of Muste et al., when we use the PTFM and the CTFM, respectively. In Fig. 12, we used the value of $\sigma=0.56$ for all turbulence models, and noted that while the results agreed with the 


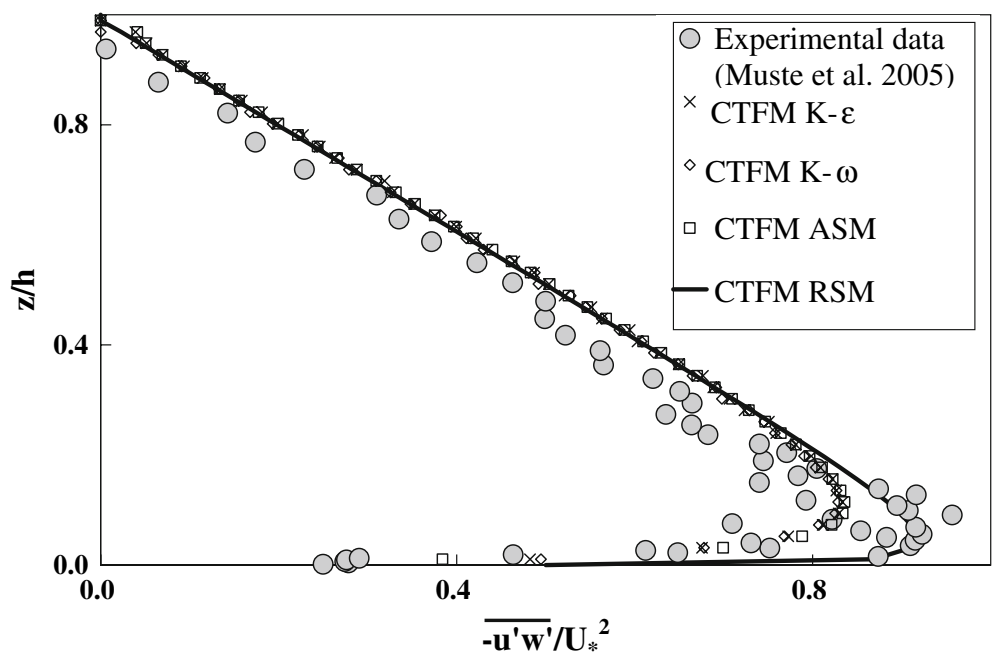

Fig. 11 Comparison of simulated values of the non-dimensional shear stress of the carrier phase with the experimental data. Simulations were performed using the $K-\varepsilon$ and $K-\omega$ models, the ASM and the RSM, under the approach of the CTFM

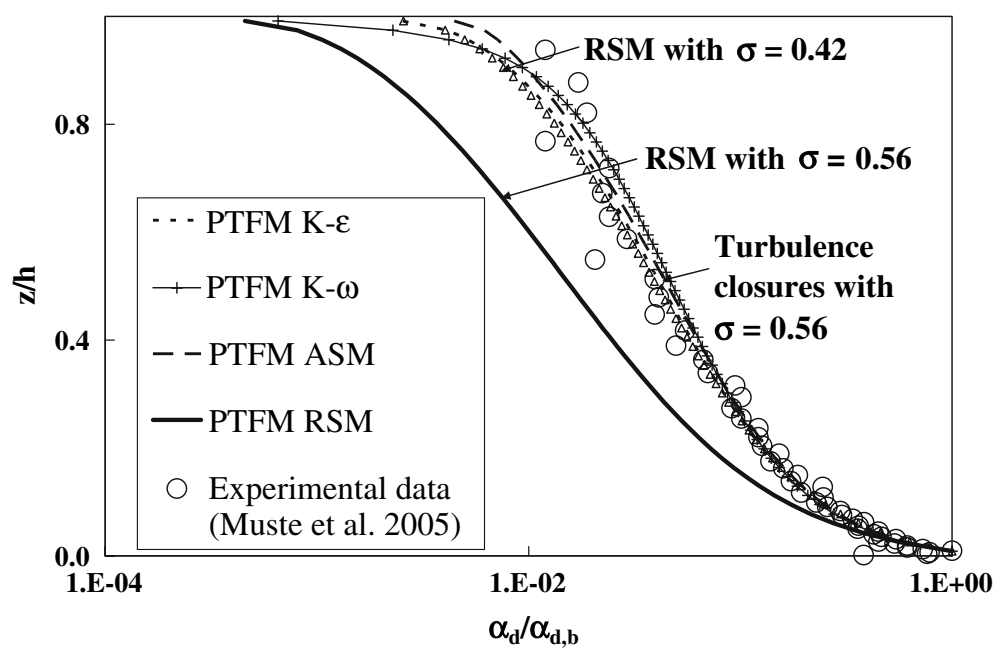

Fig. 12 Comparison of simulated values of the distribution of the volume fraction of the disperse phase with the experimental data. Simulations were performed using the $K-\varepsilon$ and $K-\omega$ models, the ASM and the RSM, under the approach of the PTFM

data for the $K-\varepsilon$ and the $K-\omega$ models, and the ASM, the results with the RSM deviated from the data. Only when the value of the Schmidt number was reduced to 0.42 , the RSM provided a good fit. Interestingly, the use of $\sigma=0.56$ with the CTFM (Fig. 13) did not provide good results for any of the turbulence closures. Using now $\sigma=0.4$, all models except the RSM provided good predictions. Only when the value of the Schmidt number was reduced to 0.3 in the CTFM, the RSM gave satisfactory predictions. 


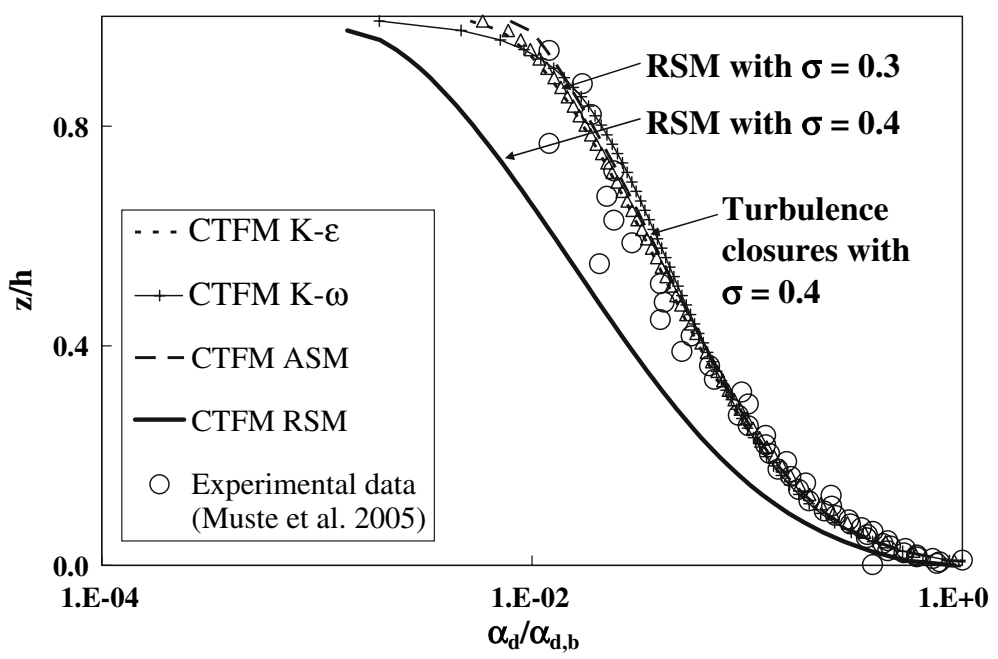

Fig. 13 Comparison of simulated values of the distribution of the volume fraction of the disperse phase with the experimental data. Simulations were performed using the $K-\varepsilon$ and $K-\omega$ models, the ASM and the RSM, under the approach of the CTFM

We investigated this issue further via more comparisons of model predictions with the distribution of sediment in the wall-normal direction and the datasets of $[19,53,69]$. In Fig. $14 \mathrm{a}-\mathrm{c}$, we show that, in all cases, the values of $\sigma$ that facilitate the agreement with the data are consistently lower for the runs with the RSM than those with the $K-\varepsilon$ model. Also, the values of $\sigma$ estimated using the CTFM were found to be mostly smaller than those obtained using the PTFM. (The values of the Schmidt number pertaining to each case are displayed on Table 5.) In Fig. 15, we plotted the values of $\sigma$ obtained from the PTFM and the CTFM for all test cases, together with values computed using the van Rijn's [68] formula, the Einstein and Chien model [24], and laboratory observations on which van Rijn himself based his formula [68]. This plot adapts a figure presented by Lyn [46]. It is possible to see in first place that the computed values of $\sigma$ lie within the ranges of experimental values reported by different authors (within a large scatter) and that model results follow the experimental trend of decreasing $\sigma$ with larger values of $\frac{W_{s}}{U_{*}}$. In second place, these experimental values can be as small as 0.3 . Third, we observe that the values of $\sigma$ obtained from runs with the $K-\varepsilon$ model are closer to the computed values using the van Rijn's formula than those obtained from the runs with the RSM. Fourth, it becomes clear that the van Rijn's expression gives the lowest values of $\sigma$ for larger ratios $\frac{W_{s}}{U_{*}}$ than the numerical results.

In order to assess the differences between the $\sigma$ values provided by diverse runs, we investigated the computed $W_{s}=\left|W_{d}\right|$ and $U_{*}$. We found that relatively small differences in $W_{s}$ and $U_{*}$ (of the order of $20 \%$ or less) produce larger ratios $\frac{W_{s}}{U_{*}}$ for the CTFM, as opposed to the PTFM, and for the RSM, as opposed to the $K-\varepsilon$ model. As a consequence, larger $\frac{W_{s}}{U_{*}}$ ratios are associated with smaller values of $\sigma$ (see Table 6). In other words, the smaller values of $\sigma$ are the result of the small differences among runs in the values of the computed $W_{s}$ and $U_{*}$. (Recall that we used the reported values of $W_{s}$ in the PTFM and that these values of $W_{s}$ are not measured, which implies a range of uncertainty in the reported values.)

We carried out a regression using the values of the Schmidt number obtained from various runs. Our analysis resulted in the following: 

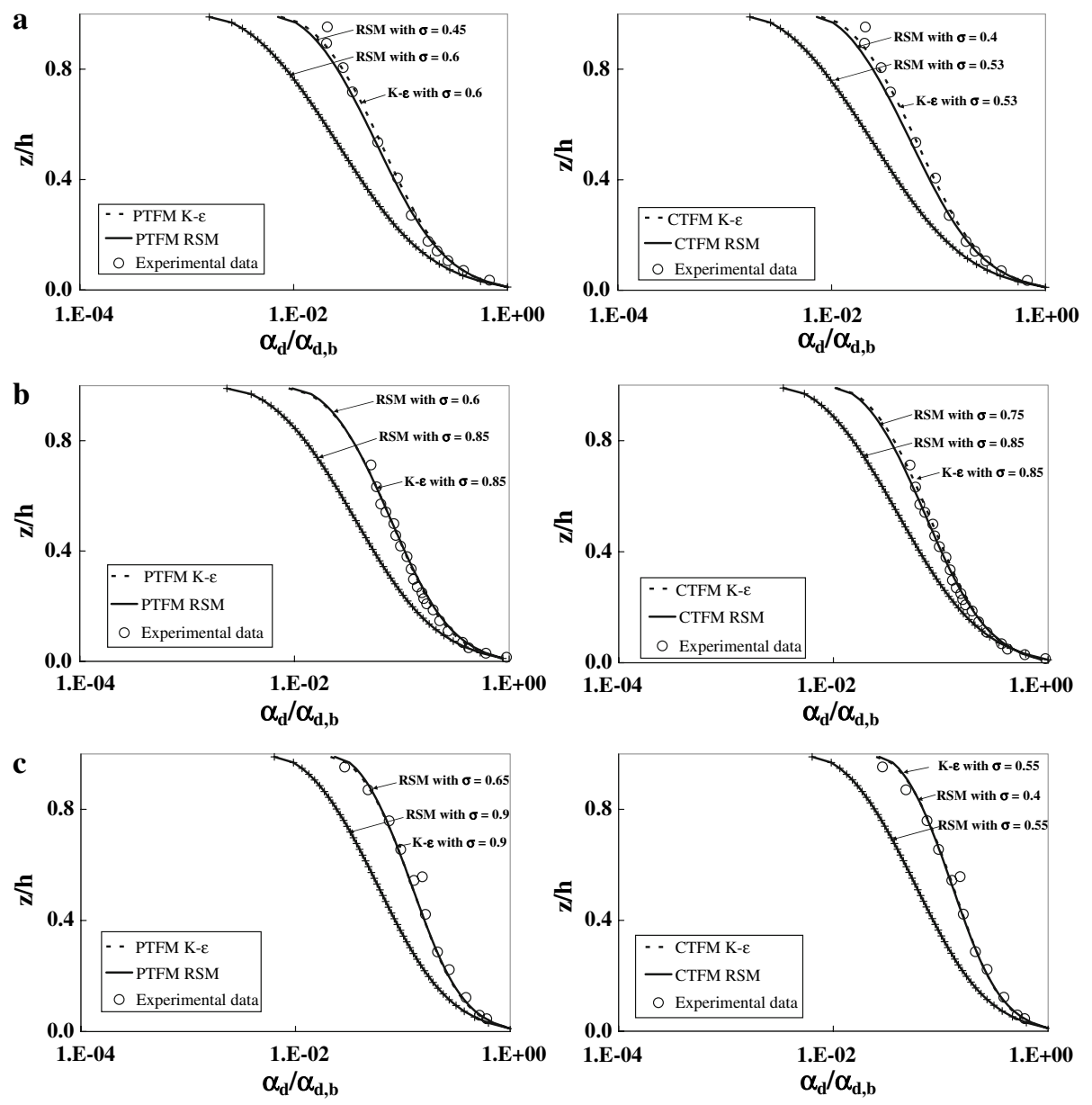

Fig. 14 a Comparison of simulated values of the distribution of the volume fraction of the disperse phase with the experimental data of [19, test case 23] b Comparison of simulated values of the distribution of the volume fraction of the disperse phase with the experimental data of [53, test case PS05]. $\mathbf{c}$ Comparison of simulated values of the distribution of the volume fraction of the disperse phase with the experimental data of [69, test case 18$]$

Table 5 Summary of the values of the Schmidt number obtained from various runs

\begin{tabular}{llllll}
\hline $\begin{array}{l}\text { Reference } \\
\text { data source }\end{array}$ & Case & $\begin{array}{l}\sigma \text { fitting } \\
\text { data } K-\varepsilon \\
(\text { PTFM) }\end{array}$ & $\begin{array}{l}\sigma \text { fitting } \\
\text { data } K-\varepsilon \\
(\text { CTFM })\end{array}$ & $\begin{array}{l}\sigma \text { fitting } \\
\text { data RSM } \\
(\text { PTFM) }\end{array}$ & $\begin{array}{l}\sigma \text { fitting } \\
\text { data RSM } \\
(\text { CTFM })\end{array}$ \\
\hline$[69]$ & 18 & 0.9 & 0.55 & 0.65 & 0.4 \\
{$[19]$} & 19 & 0.9 & 0.85 & 0.65 & 0.65 \\
& 23 & 0.6 & 0.53 & 0.45 & 0.4 \\
{$[53]$} & 26 & 0.62 & 0.53 & 0.45 & 0.39 \\
{$[51]$} & 29 & 0.7 & 0.65 & 0.5 & 0.45 \\
& PS05 & 0.85 & 0.85 & 0.6 & 0.75 \\
& NS1 & 0.56 & 0.4 & 0.42 & 0.3 \\
\hline
\end{tabular}




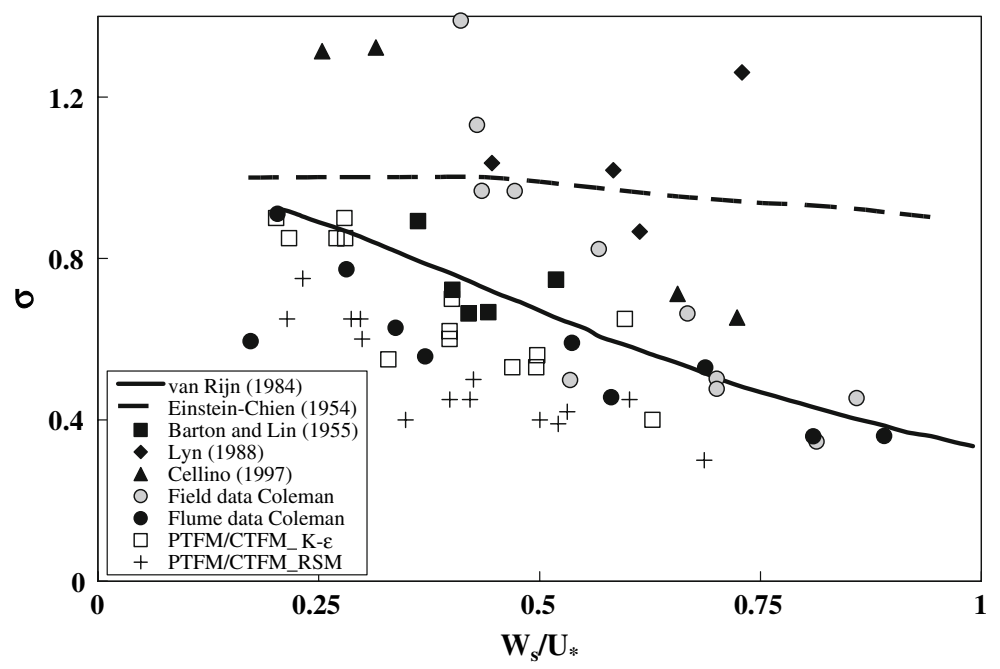

Fig. 15 Comparison of values of the Schmidt number obtained from our runs with the $K-\varepsilon$ model and the RSM (under the framework models of the PTFM and the CTFM); and those obtained from observations (adapted from [46]). The van Rijn formula and the Einstein and Chien model are also included

Table 6 Settling velocity and shear velocity obtained from the numerical models for the test case of [51]

\begin{tabular}{llll}
\hline Run & $W_{S}(\mathrm{~m} / \mathrm{s})$ & $U_{*}(\mathrm{~m} / \mathrm{s})$ & $\frac{W_{S}}{U_{*}}$ \\
\hline 5 & 0.024 & 0.048 & 0.498 \\
8 & 0.024 & 0.045 & 0.533 \\
12 & 0.029 & 0.047 & 0.611 \\
15 & 0.030 & 0.044 & 0.682 \\
\hline
\end{tabular}

$$
\sigma=\frac{1}{1.3+3\left(\frac{W_{s}}{U_{*}}\right)^{2}} \text { for } 0.1<\frac{W_{s}}{U_{*}}<1
$$

It is worth mentioning here that some authors have reported variations of $\sigma$ with the von Kármán constant $[65,71]$. In this study, the von Kármán constant is only used to impose the no-slip condition in the bottom.

It is worth also mentioning that the values of the Schmidt number do affect the velocity profiles for the CTFM. The profiles of mean velocities presented in the paper correspond to those values of the Schmidt number for which the predicted sediment volume concentrations fit the data.

6.5 Assessment of the boundary condition at the free surface, and of potential stratification effects

A physically-based, complete boundary condition for the sediment can be expressed mathematically as:

$$
\left.\left(\alpha_{d} W_{s}+D_{d} \frac{\partial \alpha_{d}}{\partial z}\right)\right|_{z=h}=0
$$

where $D_{d}$ is obtained from the turbulence model. 

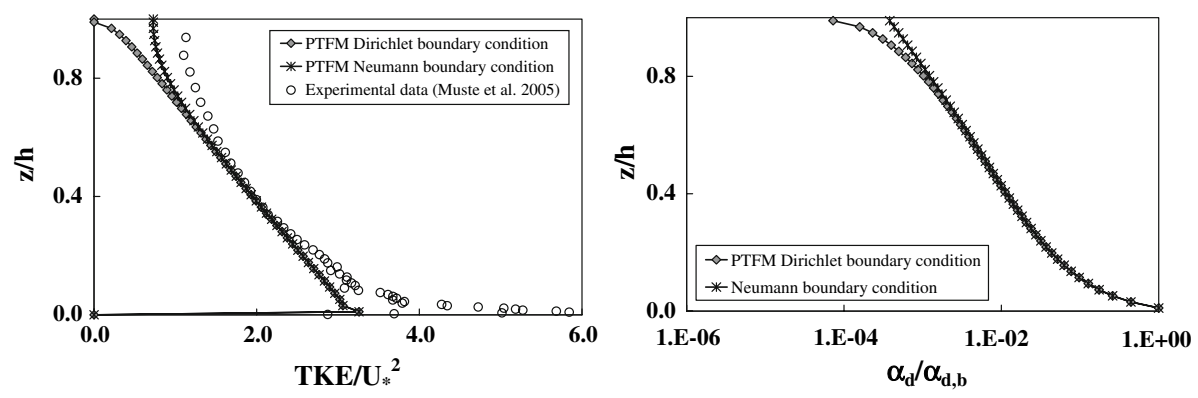

Fig. 16 Assessment of the boundary condition at the free surface. Simulations were performed using the $K-\varepsilon$ model, under the approach of the PTFM

To satisfy Eq. 9, we considered two possible alternatives: (a) $\left.\alpha_{d}\right|_{z=h}=0$, and $\left.D_{d}\right|_{z=h}=0$; (b) $\left.W_{s}\right|_{z=h}=0$; and $\left.\frac{\partial \alpha_{d}}{\partial z}\right|_{z=h}=0$. For alternative (a), in order to make $D_{d}$ zero, we imposed the condition on TKE as $\left.K\right|_{z=h}=0$ (see [6]); we called this alternative the Dirichlet boundary condition. For alternative (b), we imposed $\left.\frac{\partial K}{\partial z}\right|_{z=h}=0$; we called this alternative the Neumann boundary condition. In Fig. 16, we present a comparison of predictions of the TKE and $\alpha_{d}$ obtained with both alternative approaches. The results presented in Fig. 16 were obtained with the models of Run 5. By comparing the performance of the two approaches in Fig. 16, we observe that there is an improvement in the prediction of the TKE by applying alternative (b), but $\alpha_{d}$ takes a relatively large value which does not seem realistic in the case of dilute flows. That is why we chose to use alternative (a) throughout the present study. This result points to the fact that the boundary condition at the free surface is somewhat uncertain.

In order to corroborate that stratification effects are really minor in our selected experimental tests, we computed the correction factor proposed by Gelfenbaum and Smith [26] (see [54]) as:

$$
F_{2}(R i)=\frac{1}{1+10 X}
$$

where $X=\frac{1.35 R i}{1+1.35 R i}$ and $R i$ is the Richardson number. We computed the Richardson number as follows [54]:

$$
R i=R g \frac{\frac{d C}{d z}}{\left(\frac{d U_{c}}{d z}\right)^{2}}
$$

where $R=\frac{\rho_{d}}{\rho_{c}}-1 ; U_{c}=U_{c}(z)$; and $C=C(z)$. Using the values of $C=C(z)$ and $U_{c}=U_{c}(z)$ obtained from simulation runs, we were able to determine that $F_{2}(R i) \approx 1$, which means that no stratification correction is needed for the tests analyzed in this paper.

\section{Conclusions}

In this manuscript, we have followed a rigorous approach to assess the prediction capability of different turbulence closures in the simulation of dilute sediment-laden, open-channel flows. To that end, we presented extensions of the RSM and of the $K-\omega$ model. While we adopted similar steps to those followed by other authors to obtain the extended RSM, we have proposed herein what we believe is the first $K-\omega$ for two-phase flows. Our numerical results show that: 
(1) In order to overcome the limitations of the Rousean distribution for the prediction of dilute sediment transport in suspension, the PTFM in combination with the $K-\varepsilon$ model provides satisfactory results (accuracy of the order of 5-10\%). The values of the Schmidt number required for the agreement of sediment volume fractions with data are close to the predictions using the van Rijn expression, although slightly smaller. The use of alternative turbulence models such as the ASM and the $K-\omega$ model together with the PTFM also provides satisfactory results, albeit for different Schmidt numbers. (These Schmidt numbers are also smaller than those provided by the van Rijn formula.)

(2) The use of the PTFM for dilute mixtures paired with different turbulence closures $(K-\varepsilon$ and $K-\omega$ models and ASM) offers predictions of the same accuracy of the CTFM when paired with the same turbulence closures. All predictions with different closures are within $10 \%$ of the observations for five different datasets. In addition, the CTFM requires smaller values of the Schmidt number than the PTFM. Given the additional complexity of the CTFM, its use should be reserved mostly for non-dilute conditions.

(3) The RSM does not improve the predictions beyond the accuracy of the $K-\varepsilon$ model, for instance, whether it is paired with the PTFM or the CTFM. This result extends to two-phase flows the well-known results of single-phase flows in open channels. Additionally, the closure can be associated with smaller Schmidt numbers than the $K-\varepsilon$ model, for both the PTFM and the CTFM. Therefore, relatively simple closures such as the $K-\varepsilon$ model are able to capture the physics of the problem.

(4) As stated above, the CTFM and RSM are associated with smaller values of the Schmidt number. This is due to the fact that those models predict larger values of $W_{s}$ and a smaller values of $U_{*}$ as opposed to those obtained with the PTFM and the $K-\varepsilon$ model. We recall herein that the PTFM uses the value of $W_{s}$ reported in the experimental papers. This is an interesting issue since the trends predicted by our models agree notably with the trends in the van Rijn formula. Larger particles and/or less intense flows are associated with lower values of $\sigma$.

(5) For all tests developed, including different formulations and turbulence closures, the Schmidt number is lower than one, which emphasizes that the diffusivity of momentum is smaller than that of sediment for dilute mixtures.

(6) We have proposed an alternative expression for $\sigma$ based on our numerical computations. This regression lies within the scatter of the data.

Ongoing work includes the analysis of this framework for mixture of various sediment sizes and for non-dilute conditions.

Acknowledgments We are grateful for fruitful discussions with Prof. W. Kollmann, from the Department of Mechanical and Aeronautical Engineering, University of California, Davis, on diverse aspects of the turbulence closures. This paper was completed thanks to the support of the University of California Water Resources Center (UC WRC) and the Cooperative Institute for Coastal and Estuarine Environment Technology (CICEET). This support is gratefully acknowledged.

Open Access This article is distributed under the terms of the Creative Commons Attribution Noncommercial License which permits any noncommercial use, distribution, and reproduction in any medium, provided the original author(s) and source are credited.

\section{Appendix}

In order to obtain the transport equations for the stresses, we start from the basic two-phase flow equations, i.e., the two-fluid model, TFM [21]. By assuming incompressibility and no 
phase change (similar to steps taken by [Kataoka and Serizawa 1989, p. 846; 38 and 48]), the governing equations can be written as follows:

Continuity equation

$$
\frac{\partial U_{j, k}}{\partial x_{j}}=0
$$

Momentum equations

$$
\frac{\partial U_{i, k}}{\partial t}+\frac{\partial}{\partial x_{j}}\left(U_{i, k} U_{j, k}\right)=-\frac{1}{\rho_{k}} \frac{\partial P_{k}}{\partial x_{i}}+\frac{1}{\rho_{k}} \frac{\partial}{\partial x_{j}}\left(T_{i j, k}+T_{i j, k}^{E A}\right)+\frac{M_{i}}{\alpha_{k} \rho_{k}} \pm \frac{\beta U_{i, r}}{\alpha_{k} \rho_{k}}
$$

where $T_{i j, k}$ refers to the deviatoric stresses; and the stresses with the superscript $E A$ are the result of the process of ensemble averaging.

We decompose the variables in Eqs. A.1 and A.2 into time-averaged components (upper case letters with overbar), and fluctuations (lower case letters with the superscript) as follows:

$$
\begin{gathered}
\alpha_{k}=\bar{\alpha}_{k}+\alpha_{k}^{\prime} ; \quad U_{i, k}=\overline{U_{i, k}}+u_{i, k}^{\prime} ; \quad P_{k}=\overline{P_{k}}+p_{k}^{\prime} ; \quad T_{i j, k}=\overline{T_{i j, k}}+t_{i j, k}^{\prime} ; \\
T_{i j, k}^{E A}=\overline{T_{i j . k}^{E A}}+t_{i j, k}^{E A^{\prime}} ; \quad \beta=\bar{\beta}+\beta^{\prime} ; \quad M_{i}=\overline{M_{i}}+M_{i}^{\prime}
\end{gathered}
$$

On introducing variables defined in Eq. A.3, the following equations result:

$$
\begin{gathered}
\frac{\partial\left(\overline{U_{i, k}}+u_{i, k}^{\prime}\right)}{\partial x_{j}}=0 \\
\frac{\partial\left(\overline{U_{i, k}}+u_{i, k}^{\prime}\right)}{\partial t}+\frac{\partial}{\partial x_{j}}\left[\left(\overline{U_{i, k}}+u_{i, k}^{\prime}\right)\left(\overline{U_{j, k}}+u_{j, k}^{\prime}\right)\right]=-\frac{1}{\rho_{k}} \frac{\partial\left(\overline{P_{k}}+p_{k}^{\prime}\right)}{\partial x_{i}} \\
+\frac{1}{\rho_{k}} \frac{\partial}{\partial x_{j}}\left(\overline{T_{i j, k}}+t_{i j, k}^{\prime}+\overline{T_{i j, k}^{E A}}+t_{i j, k}^{E A^{\prime}}\right)+\frac{\overline{M_{i}}+M_{i}^{\prime}}{\alpha_{k} \rho_{k}} \pm \frac{\left(\bar{\beta}+\beta^{\prime}\right)\left(\bar{U}_{i, r}+u_{i, r}^{\prime}\right)}{\alpha_{k} \rho_{k}}
\end{gathered}
$$

We then perform time averaging on Eqs. A.4 and A.5. The final averaged equations take the form as follows:

$$
\begin{aligned}
& \frac{\partial\left(\overline{U_{j, k}}\right)}{\partial x_{j}}=0 \\
& \frac{\partial \overline{U_{i, k}}}{\partial t}+\frac{\partial}{\partial x_{j}}\left(\overline{U_{i, k}} \overline{U_{j, k}}+\overline{u_{i, k}^{\prime} u_{j, k}^{\prime}}\right)=-\frac{1}{\rho_{k}} \frac{\partial \overline{P_{k}}}{\partial x_{i}}+\frac{1}{\rho_{k}} \frac{\partial}{\partial x_{j}}\left(\overline{T_{i j, k}}+\overline{T_{i j, k}^{E A}}\right) \\
& +\frac{\overline{M_{i}}}{\alpha_{k} \rho_{k}} \pm \frac{\left(\overline{\beta U_{i, r}}+\overline{\beta^{\prime} u_{i, r}^{\prime}}\right)}{\alpha_{k} \rho_{k}}
\end{aligned}
$$

(In the above equations, we approximated the time average of the fluctuations of the forces as zero.) On subtracting Eqs. A.6 and A.7 from Eqs. A.4 and A.5, respectively, we obtain the fluctuation equations as:

$$
\frac{\partial\left(u_{i, k}^{\prime}\right)}{\partial x_{j}}=0
$$




$$
\begin{gathered}
\frac{\partial\left(u_{i, k}^{\prime}\right)}{\partial t}+\frac{\partial}{\partial x_{j}}\left[\left(\overline{U_{i, k}} u_{j, k}^{\prime}+\overline{U_{j, k}} u_{i, k}^{\prime}+u_{i, k}^{\prime} u_{j, k}^{\prime}-\overline{u_{i, k}^{\prime} u_{j, k}^{\prime}}\right)\right]=-\frac{1}{\rho_{k}} \frac{\partial p_{k}^{\prime}}{\partial x_{i}} \\
+\frac{1}{\rho_{k}} \frac{\partial}{\partial x_{j}}\left(t_{i j, k}^{\prime}+t_{i j, k}^{E A^{\prime}}\right)+\frac{M_{i}^{\prime}}{\alpha_{k} \rho_{k}} \pm \frac{\left(\bar{\beta} u_{i, r}^{\prime}+\beta^{\prime} \overline{U_{i, r}}+\beta^{\prime} u_{i, r}^{\prime}-\overline{\beta^{\prime} u_{i, r}^{\prime}}\right)}{\alpha_{k} \rho_{k}}
\end{gathered}
$$

The equations for $\overline{u_{i, k}^{\prime} u_{j, k}^{\prime}}$ can then be obtained after the following steps: (a) Multiplication of Eq. A.9 by $u_{j}^{\prime}$; (b) interchange of the subscripts $i$ and $j$; (c) addition of the equations obtained in steps (a) and (b) followed by time averaging; and, finally, application of the product rule to obtain the transport equation for $\overline{u_{i, k}^{\prime} u_{j, k}^{\prime}}$. Similar steps were employed by Kumar [38] and Mashayek and Taulbee [48] in their models.

Here we focus on those terms in Eq. A.9 which appear due to the inter-phase interactions (the last large term in (A.9)):

$$
\begin{aligned}
& \overline{u_{i, k}^{\prime}}\left(\bar{\beta} u_{j, r}^{\prime}+\beta^{\prime} \overline{U_{j, r}}+\beta^{\prime} u_{j, r}^{\prime}-\overline{\beta^{\prime} u_{j, r}^{\prime}}\right)+u_{j, k}^{\prime}\left(\bar{\beta} u_{i, r}^{\prime}+\beta^{\prime} \overline{U_{i, r}}+\beta^{\prime} u_{i, r}^{\prime}-\overline{\beta^{\prime} u_{i, r}^{\prime}}\right) \\
&=\bar{\beta}\left(\overline{u_{i, k}^{\prime} u_{j, r}^{\prime}}+\overline{u_{j, k}^{\prime} u_{i, r}^{\prime}}\right)+\overline{U_{j, r}} \overline{\beta^{\prime} u_{i, k}^{\prime}}+\overline{U_{i, r}} \overline{\beta^{\prime} u_{j, k}^{\prime}}+\overline{u_{i, k}^{\prime} \beta^{\prime} u_{j, r}^{\prime}}+\overline{u_{j, k}^{\prime} \beta^{\prime} u_{i, r}^{\prime}} \\
&-\overline{u_{i, k}^{\prime} \overline{\beta^{\prime} u_{j, r}^{\prime}}}-\overline{u_{j, k}^{\prime} \overline{\beta^{\prime} u_{i, r}^{\prime}}} \\
&= \bar{\beta}\left(\overline{u_{i, k}^{\prime} u_{j, r}^{\prime}}+\overline{u_{j, k}^{\prime} u_{i, r}^{\prime}}\right)+\overline{U_{j, r}} \overline{\beta^{\prime} u_{i, k}^{\prime}}+\overline{U_{i, r}} \overline{\beta^{\prime} u_{j, k}^{\prime}}+\overline{u_{i, k}^{\prime} \beta^{\prime} u_{j, r}^{\prime}}+\overline{u_{j, k}^{\prime} \beta^{\prime} u_{i, r}^{\prime}}
\end{aligned}
$$

In what follows, we neglect the third-order correlations in (A.10) as performed in previous papers. Mashayek and Taulbee [48] stated that these higher-order terms are usually small, and that their effects may be absorbed in the pressure terms (p. 36). Thus Eq. A.10 takes the following form:

$$
=\bar{\beta}\left(\overline{u_{i, k}^{\prime} u_{j, r}^{\prime}}+\overline{u_{j, k}^{\prime} u_{i, r}^{\prime}}\right)+\overline{U_{i, r}} \overline{\beta^{\prime} u_{j, k}^{\prime}}+\overline{U_{j, r}} \overline{\beta^{\prime} u_{i, k}^{\prime}}
$$

Finally, extending the RSM for two-phase flows leads to the following transport equation for the Reynolds stresses of the carrier phase (please note that we have removed overbars from the mean variables for simplicity), where terms appear premultiplied by the volume fraction of the carrier:

$$
\begin{aligned}
\frac{D}{D t}\left[\alpha_{c} \rho_{c}\left(\overline{u_{i, c}^{\prime} u_{j, c}^{\prime}}\right)\right]= & P_{i j}+D_{i j}+\pi_{i j}-\varepsilon_{i j}-\beta\left(\overline{\left(u_{j, c}^{\prime} u_{i, r}^{\prime}\right.}+\overline{u_{i, c}^{\prime} u_{j, r}^{\prime}}\right) \\
& -U_{i, r} \overline{\beta^{\prime} u_{j, c}^{\prime}}-U_{j, r} \overline{\beta^{\prime} u_{i, c}^{\prime}}
\end{aligned}
$$

where

$$
\beta^{\prime}=\frac{3}{4 d_{p}} \alpha_{d}^{\prime} \rho_{c} C_{D}\left|\vec{U}_{r}\right|
$$

The third-to-last term of Eq. A.12 can be expanded to:

$$
S_{u_{i} u_{j}, 1}=\beta\left[\overline{\left[u_{j, c}^{\prime} u_{i, r}^{\prime}\right.}+\overline{u_{i, c}^{\prime} u_{j, r}^{\prime}}\right]=\beta\left[\overline{u_{j, c}^{\prime}\left(u_{i, c}^{\prime}-u_{i, d}^{\prime}\right)}+\overline{u_{i, c}^{\prime}\left(u_{j, c}^{\prime}-u_{j, d}^{\prime}\right)}\right]
$$

The right hand side of Eq. A.14 contains terms like $\overline{u_{i, c}^{\prime} u_{j, c}^{\prime}}$ which are the Reynolds stresses. Besides, there are terms of the form $\overline{u_{j, c}^{\prime} u_{i, d}^{\prime}}$ and $\overline{u_{i, c}^{\prime} u_{j, d}^{\prime}}$ (representing the correlation between the turbulent velocity fluctuations of the two phases) which require further closure. Several 
approaches have been used recently to model these correlations. The most general (but numerically most costly) approach is to derive transport equations for these quantities, but these equations require even further closure due to the appearance of more correlations of triple order (see comment above). This approach was proposed by Mashayek and Taulbee [48] in their derivation of an explicit algebraic model from a general two-phase RSM. On the other hand, Lian and Aliod [39] expressed this type of correlations in terms of the Reynolds stresses of the disperse phase. It is worth pointing out that recent approaches have simplified this type of correlations by writing them in terms of the TKE of the carrier phase (see [17]). In this work, we applied the closure proposed by Ma and Ahmadi [47] as follows:

$$
\overline{u_{j, c}^{\prime} u_{i, d}^{\prime}}=f_{u} \overline{u_{i, c}^{\prime} u_{j, c}^{\prime}}
$$

where $f_{u}=\frac{1}{1+T_{P} / T_{L}}$, and the particle $\left(T_{P}\right)$ and fluid time $\left(T_{L}\right)$ scales are defined as:

$$
T_{p}=\frac{\rho_{d}}{\frac{3}{4 d_{p}} \rho_{c} C_{D} \mid \overrightarrow{U_{r} \mid}} ; \quad \text { and } \quad T_{L}=0.165 \frac{K}{\varepsilon} .
$$

This expression by Ma and Ahmadi [47] was developed for gas-solid flows. It is a function of particle and flow time scales, including the respective densities for the carrier and the disperse phases. Since there are no similar experiences for water-solid flows, we used those expressions directly, considering the appropriate densities of water and sediment particles.

The last two terms on the right hand side of Eq. A.12 can be expanded as follows:

$$
\begin{aligned}
S_{u_{i} u_{j}, 2} & =U_{i, r} \overline{\beta^{\prime} u_{j, c}^{\prime}}+U_{j, r} \overline{\beta^{\prime} u_{i, c}^{\prime}} \\
& =\frac{3}{4 d_{p}} \rho_{c} C_{D}\left|\vec{U}_{r}\right|\left[\left(U_{i, c}-U_{i, d}\right) \overline{\alpha_{d}^{\prime} u_{j, c}^{\prime}}+\left(U_{j, c}-U_{j, d}\right) \overline{\alpha_{d}^{\prime} u_{i, c}^{\prime}}\right]
\end{aligned}
$$

In this equation, the right-hand side contains the Reynolds fluxes, i.e., the correlation of fluctuations of the volume fraction of the disperse phase and the velocity of the carrier. A transport equation could be derived for these fluxes [48]. Considering the complexities involved in solving a large set of equations, and the scarcity of knowledge about closure of further correlations which appear in the transport equations, we applied a closure based on the Boussinesq model as $-\overline{\alpha_{d}^{\prime} u_{j, c}^{\prime}}=D_{d}\left(\partial \overline{\alpha_{d}} / \partial x_{j}\right)$, where $D_{d}$ is the diffusivity of the disperse phase, determined as follows:

$$
D_{d}=\frac{C_{\mu}}{\sigma}\left(\frac{K^{2}}{\varepsilon}\right)
$$

Including all closures discussed above into Eq. A.12 we obtain the final expression:

$$
\begin{aligned}
\frac{D}{D t}\left[\alpha_{c} \rho_{c}\left(\overline{u_{i, c}^{\prime} u_{j, c}^{\prime}}\right)\right]= & P_{i j}+D_{i j}+\pi_{i j}-\varepsilon_{i j}-2 \beta\left(1-f_{u}\right) \overline{u_{i, c}^{\prime} u_{j, c}^{\prime}} \\
& +\frac{3}{4 d_{p}} C_{D}\left|\vec{U}_{r}\right|\left[U_{i, r} D_{d} \frac{\partial \alpha_{d}}{\partial x_{j}}+U_{j, r} D_{d} \frac{\partial \alpha_{d}}{\partial x_{i}}\right]
\end{aligned}
$$

\section{References}

1. Beishuizen NA, Naud B, Roekaerts D (2007) Evaluation of a modified Reynolds stress model for turbulent dispersed two-phase flows including two-way coupling. Flow Turbul Combust 79:321-341

2. Bertodano ML, Saif AA (1997) Modified $k-\varepsilon$ model for two-phase turbulent jets. Nucl Eng Des 172:187196 
3. Bertodano ML, Lee S-J, Lahey RT, Drew DA (1990) The prediction of two-phase turbulence and phase distribution phenomena using a Reynolds stress model. J Fluids Eng 112:107-113

4. Bombardelli FA (2004) Turbulence in multiphase models for aeration bubble plumes. PhD Thesis. University of Illinois at Urbana-Champaign, Department of Civil and Environmental Engineering

5. Bombardelli FA, Gioia G (2006) Scouring of granular beds by jet-driven axisymmetric turbulent cauldrons. Phys Fluids 18:088101

6. Bombardelli FA, Jha SK (2008) Hierarchical modeling of dilute, suspended-sediment transport in open channels. Environ Fluid Mech. doi:10.1007/s10652-008-9091-6

7. Bombardelli FA, Buscaglia GC, García MH (2003) Parallel computations of the dynamic behavior of bubble plumes. In: Brust FW (ed) Proceedings of the pressure vessels and pipe division conference, Cleveland, vol PVP-464, Residual Stress, Fitness-for-Service, and Manufacturing Processes. ASME-PVP Division

8. Brennen CE (2005) Fundamentals of multiphase flow. Cambridge University Press, Cambridge

9. Buscaglia GC, Bombardelli FA, García MH (2002) Numerical modeling of large scale bubble plumes accounting for mass transfer effects. Int J Multiph Flow 28:1763-1785

10. Cao Z, Carling PA (2002) Mathematical modeling of alluvial rivers: reality and myth. Part 2: special issues. Proc Inst Civ Eng Water Marit Eng 154(4):297-308

11. Cao Z, Wei L, Xie J (1995) Sediment-laden flow in open channels from two-phase flow viewpoint. J Hydraul Eng 121(10):725-735

12. Chauchat J (2007) Contribution to two-phase flow modeling for sediment transport in estuarine and coastal zones. PhD Thesis, University of Caen, France (in French)

13. Chauchat J, Guillou S (2008) On turbulence closures for two-phase sediment-laden flow models. J Geophys Res 113(C11017)

14. Chen CP, Wood PE (1986) Turbulence closure modeling of the dilute gas-particle axisymmetric jet. AIChe J 32(1):163-166

15. Choi S, Kang H (2004) Reynolds stress modeling of vegetated open-channel flows. J Hydraul Res 42(1):3-11

16. Cokljat D, Younis BA (1995) Second order closure study of open-channel flows. J Hydraul Eng 121(2):94-107

17. Cokljat D, Ivanov VA, Sarasola FJ, Vasquez SA (2000) Multiphase k-epsilon models for unstructured meshes. In: ASME 2000 Fluids Engineering Division Summer Meeting, Boston

18. Cokljat D, Slack M, Vasquez SA, Bakker A, Montante G (2006) Reynolds-stress model for Eulerian multiphase. In: Nagano Y, Hanjalic K, Tummers MJ (eds) Proceedings of the 4th international symposium on turbulence heat and mass transfer, pp 1047-1054

19. Coleman NL (1986) Effects of suspended sediment on the open-channel distribution. Water Resour Res 22(10): $1377-1384$

20. Drew DA (1975) Turbulent sediment transport over a flat bottom using momentum balance. J Appl Mech, Trans ASME 42:38-44

21. Drew D, Passman S (1999) Theory of multicomponent fluids. Applied mathematical sciences. Springer, Berlin

22. Elghobashi S (1994) On predicting particle-laden turbulent flows. Appl Sci Res 52:309-329

23. Elghobashi S, Abou-Arab TW (1983) A two-equation turbulence model for two-phase flows. Phys Fluids 26(4):931-938

24. Einstein HA, Chien N (1955) Effects of heavy sediment concentration near the bed on velocity and sediment distribution. MRD Sediment Series Report No. 8, University of California, Berkeley, U.S. Army Corps of Engineers, Missouri Division

25. Gatski TB, Speziale CG (1993) On explicit algebraic stress models for complex turbulent flows. J Fluid Mech 254:59-78

26. Gelfenbaum G, Smith JD (1986) Experimental evaluation of a generalized suspended-sediment transport theory. In: Knight RJ, McLean JR (eds) Shelf and sandstones. Canadian Society of Petroleum Geologists Memoir II. pp 133-144

27. Gioia G, Bombardelli FA (2002) Scaling and similarity in rough channel flows. Phys Rev Lett 88(1):014501

28. Gioia G, Bombardelli FA (2005) Localized turbulent flows on scouring granular beds. Phys Rev Lett 95:014501

29. Gioia G, Chakraborty P, Bombardelli FA (2006) Rough-pipe flows and the existence of fully developed turbulence. Phys Fluids 18:038107

30. Greimann BP, Holly FM Jr (2001) Two-phase flow analysis of concentration profiles. J Hydraul Eng 127(9):753-762

31. Greimann BP, Muste M, Holly FM Jr (1999) Two-phase formulation of suspended sediment transport. J Hydraul Res 37:479-500 
32. Hsu T, Jenkins JT, Liu PLF (2003) On two-phase sediment transport: dilute flow. J Geophys Res 108(C3):3057

33. Jaw SY, Chen CJ (1998) Present status of second order closure turbulence models. II Applications. J Eng Mech 124(5):502-512

34. Jiang J, Law AW, Cheng NS (2004) Two-phase analysis of vertical sediment laden jets. J Eng Mech 131(3):308-318

35. Kang H, Choi S-Uk (2006) Reynolds stress modeling of rectangular open-channel flow. Int J Numer Methods Fluids 51:1319-1334

36. Kataoka I, Serizawa A (1989) Basic equations of turbulence in gas-liquid two-phase flow. Int J Multiph Flow 15(5), 843-885

37. Kobayashi N, Seo SN (1985) Fluid and sediment interaction over a plane bed. J Hydraul Eng 111(6):903919

38. Kumar R (1995) An algebraic stress/flux model for two-phase turbulent flow. In: Second ISHMIT-ASME heat and mass conference, Karnataka, India, 28-30 Dec

39. Laín S, Aliod R (2003) Discussion on second-order dispersed phase Eulerian equations applied to turbulent particle-laden jet flows. Chem Eng Sci 58:4527-4535

40. Launder BE, Reece GJ, Rodi W (1975) Progress in the development of a Reynolds-stress turbulent closure. J Fluid Mech 68(3):537-566

41. Lien FS, Leschzinger MA (1994) Assessment of turbulence-transport models concluding non-linear RNG eddy-viscosity formulation and second-moment closure for flow over a backward-facing step. Comput Fluids. 23(8), 983-1004

42. López F, García MH (1998) Open-channel flow through simulated vegetation: suspended sediment transport modeling. Water Resour Res 34(9):2341-2352

43. López F, García M (2001) Mean flow and turbulence structure of open-channel flow through non-emergent vegetation. J Hydraul Eng 127(5):392-402

44. Loth E (2007) Computational fluid dynamics of bubbles, drops and particles. Cambridge University Press, Cambridge

45. Lyn DA (1988) A similarity approach to turbulent sediment-laden flows in open channels. J Fluid Mech 193:1-26

46. Lyn DA (2008) Sedimentation engineering: theories, measurements, modeling and practice. In: García M (ed) Manual No. 110, ASCE, 1150 pp

47. Ma D, Ahmadi G (1988) A kinetic model for rapid granular flows of nearly elastic particles including interstitial fluid effects. Powder Technol 56:191-207

48. Mashayek F, Taulbee DB (2002) A four-equation model for prediction of gas-solid turbulent flows. Numer Heat Transf 41:95-116

49. McTigue DF (1981) Mixture theory for suspended sediment transport. J Hydraul Div 107(HY6): 659-673

50. Muste M, Patel VC (1997) Velocity profiles for particles and liquid in open-channel flow with suspended sediment. J Hydraul Eng 123(9):742-751

51. Muste M, Fujita K, Yu I, Ettema R (2005) Two-phase versus mixed-flow perspective on suspended sediment transport in turbulent channel flows. Water Resour Res 41:W10402

52. Nezu I (2005) Open-channel flow turbulence and its research prospect in the 21 st century. J Hydraul Eng 131(4):229-246

53. Nezu I, Azuma R (2004) Turbulence characteristics and interaction between particles and fluid in particle-laden open-channel flows. J Hydraul Eng 130:988-1001

54. Parker G (2004) 1D sediment transport morphodynamics with application to rivers and turbidity currents. e-book downloadable at: http://cee.uiuc.edu/people/parkerg/morphodynamics_ebook.htm

55. Parthasarathy RN, Faeth GM (1987) Structure of particle-laden turbulent water jets in still water. Int J Multiph Flow 13(5):699-716

56. Pope SB (1975) A more general effective viscosity hypothesis. J Fluid Mech 72(2):331-340

57. Pope SB (2000) Turbulent flows. Cambridge University Press, Cambridge

58. Rodi W (1984) Turbulence models and their application in hydraulics. International Association for Hydraulic Research, Delft, The Netherlands

59. Rubinstein R, Zhou Y, Younis BA (1997) The dissipation rate transport equation in rotating turbulent shear flow. In: Proceedings of the 13th symposium on turbulent shear flows

60. Sijercic M, Belosevic S, Stevanovic Z (2007) Simulation of free turbulent particle-laden jet using Reynolds-stress gas turbulence model. Appl Math Model 31:1001-1014

61. Sokolichin A, Eigenberger G (1999) Applicability of the standard turbulence model to the dynamic simulation of bubble columns: Part I. Detailed numerical simulations. Chem Eng Sci 54:2273-2284 
62. Sokolichin A, Eigenberger G, Lapin A (2004) Simulation of buoyancy driven bubbly flow: established simplifications and open questions. AIChe J 50:24-45

63. Speziale CG, Younis BA, Berger SA (2000) Analysis and modeling of turbulent flow in an axially rotating pipe. J Fluid Mech 407:1-26

64. Squires KD, Eaton JK (1994) Effect of selective modification of turbulence on two-equation models for particle-laden turbulent flows. J Fluid Eng 116:778-784

65. Taggart WC, Yermoli CA, Montes S, Ippen AT (1972) Effects of sediment size and gradation on concentration profiles for turbulent flow. M.I.T. Report No. 152

66. Taulbee DB, Mashayek F, Barre C (1999) Simulation and Reynolds stress modeling of particle-laden turbulent shear flows. Int J Heat Fluid Flow 20:368-373

67. Toorman EA (2008) Vertical mixing in the fully developed turbulent layer of sediment-laden open channel flow. J Hydraul Eng 134(9):1225-1235

68. Van Rijn LC (1984) Sediment transport. Part II: suspended load transport. J Hydraul Eng 110(11): 1613-1641

69. Vanoni VA (1946) Transportation of suspended sediment by water. Trans ASCE 111:67-133

70. Villaret C, Davies AG (1995) Modeling sediment-turbulent flow interactions. Appl Mech Rev 48(9):601609

71. Villaret C, Trowbridge JH (1991) Effects of stratification by suspended sediments on turbulent shear flows. J Geophys Res 96(6):10659-10680

72. Wilcox DC (1988) Reassessment of the scale-determining equation for advanced turbulence models. AIAA J 26(11):1299-1310

73. Xu Y, Subramaniam S (2006) A multiscale model for dilute turbulence gas-particle flows based on the equilibration of energy concept. Phys Fluids 18:033301-033317

74. Yoon J, Kang S (2005) A numerical model for sediment-laden turbulent flow in an open channel. Can J Civ Eng 32:233-240

75. Younis BA (1996) Progress in turbulence modeling for open-channel flows. In: Anderson MG, Walling DE, Bates PD (eds) Flood plain processes, chap 9. Wiley, New York, pp 299-332

76. Zaichik LI, Alipchenkov VM (1999) A kinetic model for the transport of arbitrary-density particles in turbulent shear flows. In: Proceedings on turbulence and shear flow phenomena 1, Santa Barbara

77. Zhou LX, Chen T (2001) Simulation of swirling gas-particle flows using USM and $k-\varepsilon-k p$ two-phase turbulence models. Powder Technol 114:1-11 